\title{
Signal propagation on $\kappa$-Minkowski spacetime and nonlocal two-point functions
}

\author{
Michele Arzano* \\ Dipartimento di Fisica "E. Pancini”, Università di Napoli Federico II, \\ Via Cinthia, 80126 Fuorigrotta, Napoli, Italy \\ and Dipartimento di Fisica, "Sapienza" Università di Roma, P.le A. Moro 2, 00185 Roma, Italy \\ Luca Tiberio Consoli ${ }^{\dagger}$ \\ Dipartimento di Fisica, "Sapienza" Università di Roma, P.le A. Moro 2, 00185 Roma, Italy
}

(Received 20 August 2018; published 26 November 2018)

\begin{abstract}
We study the propagation of quantum fields on $\kappa$-Minkowsi spacetime. Starting from the noncommutative partition function for a free field written in momentum space, we derive the Feynman propagator and analyze the nontrivial singularity structure determined by the group manifold geometry of momentum space. The additional contributions due to such a singularity structure result in a deformed field propagation which can be alternatively described in terms of an ordinary field propagation determined by a source with a blurred spacetime profile. We show that the $\kappa$-deformed Feynman propagator can be written in terms of vacuum expectation values of a commutative nonlocal quantum field. For sub-Planckian modes, the $\kappa$-deformed propagator corresponds to the vacuum expectation value of the time-ordered product of nonlocal field operators while for trans-Plankian modes, this is replaced by the Hadamard two-point function, the vacuum expectation value of the anticommutator of nonlocal field operators.
\end{abstract}

DOI: 10.1103/PhysRevD.98.106018

\section{INTRODUCTION}

A recurring theme in quantum gravity research in recent years has been that of dimensional reduction (see [1] for a comprehensive and up-to-date review). Evidence from a variety of approaches to the problem indeed suggests that the effective dimensionality of spacetime might decrease below the infrared value of four as we probe shorter and shorter scales [2-13], with the majority of results pointing to a two-dimensional effective spacetime dimension at the Planck scale.

The classical description of spacetime in terms of a smooth manifold is expected to become unreliable at very short distances, when quantum gravitational effects cannot be neglected, and thus, a notion of dimensionality in quantum gravity should be based on "dimensional estimators" [1] which can be generalized to quantum geometries. An example of such an estimator is the notion of spectral dimension associated to a diffusion process determined, via

\footnotetext{
*michele.arzano@roma1.infn.it

†consoli.1558491@studenti.uniroma1.it
}

Published by the American Physical Society under the terms of the Creative Commons Attribution 4.0 International license. Further distribution of this work must maintain attribution to the author(s) and the published article's title, journal citation, and DOI. Funded by SCOAP ${ }^{3}$. the heat equation, by a Laplacian operator. Such a characterization of dimensionality has been widely used in the literature to explore the running of dimensionality in various quantum gravity settings. The definition of spectral dimension relies on the existence of a Laplacian operator which governs the diffusion process in the particular model of quantum spacetime considered. It turns out that the nontrivial UV features of the diffusion process can be generally modeled by a deformation of the ordinary special relativistic energy-momentum dispersion relation [14]. The existence of such a departure from the usual relativistic relation between energy and momentum raises the issue of whether the phenomenon of running dimensionality could signal a breaking of Lorentz symmetry as we probe spacetime at the shortest scales [15]. Models of deformed relativistic kinematics based on curved momentum space (with a curvature scale proportional to the Planck energy $E_{p} \sim 10^{28} \mathrm{eV}$ ) provide a framework in which a running spacetime dimensionality can coexist with the notion of relativistic symmetries albeit of a deformed kind. An example of such models is given by the $\kappa$-Minkowski noncommutative spacetime [16]. The dual momentum space to this noncommutative spacetime is a non-Abelian Lie group [17] whose manifold structure is given by "half" of de Sitter space [18]. The group manifold structure of momentum space reflects on the structure of the generators 
of relativistic symmetries, which are now described by a quantum deformation of the Poincare algebra known as the $\kappa$-Poincaré algebra $[19,20]$.

A significant shortcoming of adopting the notion of a spectral dimension to gain insight on the short distance features of spacetime is that the definition of spectral dimension is based on a fictitious diffusion process on a Euclidean space [21,22]. Various alternative characterizations of the dimensionality of (quantum) spacetime in terms of estimators of more direct physical significance have appeared in recent literature [21-27]. For example, in the case of $\kappa$-Minkowski space, one can resort to field theoretic tools to describe the interaction potential between sources to gain information about the effective dimensionality of spacetime in the UV [28]. The analysis presented in [28] suggests that the nontrivial features of the Green's function leading to an effective dimensional reduction in the UV can be understood in terms of a fuzziness of the spacetime profile of the source induced by noncommutativity. In this work, we take as a starting point, this observation and embark on a systematic analysis of the construction and behavior of the Feynman propagator in $\kappa$-Minkowski space in order to gain a more complete picture of signal propagation in such a noncommutative framework.

On one side, our analysis aims to shed light on the connection between the path integral approach first pioneered in [29] and the canonical approach explored in [30] to the quantization of fields on $\kappa$-Minkowski space. The bridge between these two pictures will be established via a description of noncommutative fields on $\kappa$-Minkowski space in terms of nonlocal fields on ordinary Minkowkski space [31,32]. The work we present also aims at placing on firmer grounds the fuzzy spacetime picture of $\kappa$-deformed field propagation sketched in [28] providing an in-depth description of the spacetime properties of the $\kappa$-deformed Feynman propagator.

In the next section, we introduce the basics of $\kappa$-Minkowski noncommutative spacetime, its associated momentum space and deformed symmetries. Moreover, we collect all the necessary tools to carry out the field theoretic analysis in the following sections, namely the noncommutative differential calculus, the notion of a Weyl map, « product, and integration on noncommutative spaces. Our analysis starts in Sec. III, where we derive the $\kappa$-deformed Feynman propagator from the momentum space counterpart of the noncommutative partition function, analyze how this propagates field perturbations, and interpret the results in terms of the effects of spacetime fuzziness on sources and on the propagation process. In the remaining part of Sec. III, we study the spacetime profile of the deformed propagator function according to the various possible values of its argument. In Sec. IV, we turn to the connection between noncommutative fields on $\kappa$-Minkowski space and a nonlocal field theory on ordinary Minkowski space. In Sec. V, we proceed to a canonical quantization of the nonlocal field theory derived in the previous section and observe that the $\kappa$-deformed propagator can be obtained as the vacuum expectation value of the time-ordered product of nonlocal fields for "sub-Planckian" momenta (with a modulus lower than the deformation scale $\kappa$ ), while it coincides with the nonlocal Hadamard two-point function for trans-Planckian momenta. The concluding section is devoted to a summary and discussion of the results obtained.

\section{THE $\kappa$-DEFORMED FIELD THEORIST TOOLBOX}

The $\kappa$-Minkowski spacetime [16] is described by the four-dimensional Lie algebra

$$
\left[\hat{x}^{0}, \hat{x}^{i}\right]=\frac{i}{\kappa} \hat{x}^{i}, \quad\left[\hat{x}^{i}, \hat{x}^{j}\right]=0, \quad i, j=1,2,3 .
$$

It will be useful to work with the following five-dimensional matrix representation ${ }^{1}$ of the $\kappa$-Minkowski Lie algebra:

$$
\left(\hat{x}_{0}\right)_{a}{ }^{b}=\frac{i}{\kappa}\left(\begin{array}{ccc}
0 & \mathbf{0}^{T} & 1 \\
\mathbf{0} & \underline{\mathbf{0}} & \mathbf{0} \\
1 & \mathbf{0}^{T} & 0
\end{array}\right), \quad\left(\hat{x}_{i}\right)_{a}{ }^{b}=\frac{i}{\kappa}\left(\begin{array}{ccc}
0 & \mathbf{e}_{i}^{T} & 0 \\
\mathbf{e}_{i} & \underline{\mathbf{0}} & \mathbf{e}_{i} \\
0 & -\mathbf{e}_{i}^{T} & 0
\end{array}\right),
$$

where the 3 -vector $\mathbf{e}_{i}$ has entry 1 at the i'th position [i.e., $\mathbf{e}_{i}^{T}=\left(\delta_{i}^{1}, \delta_{i}^{2}, \delta_{i}^{3}\right)$ and $T$ denotes transposition], the 3 -vector $\mathbf{0}$ is the null vector and $\underline{0}$ is the $3 \times 3$ null matrix. Let us notice that the algebra (1) can be seen as a subalgebra of the five dimensional Lorentz algebra $\mathfrak{\mathfrak { o }}(4,1)$. Indeed, using the Iwasawa decomposition, the latter can be written as the following direct sum:

$$
\mathfrak{s} \mathfrak{o}(4,1)=\mathfrak{i} \oplus \mathfrak{a} \oplus \mathfrak{n},
$$

where $\mathfrak{f}$ is the four-dimensional Lorentz algebra $\mathfrak{g} \mathfrak{p}(3,1)$, the algebra $\mathfrak{a}$ is one-dimensional, and $\mathfrak{n}$ is a three-dimensional nilpotent algebra. A representation of the algebras $\mathfrak{a}$ and $\mathfrak{n}$ is given by the $5 \times 5$ matrices $^{2} \mathrm{a}=i \kappa\left(\hat{x}_{0}\right)_{a}{ }^{b}$ and $\mathrm{n}_{i}=i \kappa\left(\hat{x}_{i}\right)_{a}{ }^{b}$ respectively, so that they satisfy the $\kappa$-Minkowski-like commutation relation

$$
\left[\mathrm{a}, \mathrm{n}_{i}\right]=\mathrm{n}_{i},
$$

with all other commutator being zero.

Noncommutative "plane waves" are obtained by exponentiating the generators of (1). The dimensionful parameters appearing in the argument of the exponential are interpreted as $\kappa$-deformed momenta. Two important points should be stressed which radically distinguish the present

\footnotetext{
${ }^{1}$ With lower indices $\hat{x}^{0}=-\hat{x}_{0}, \quad \hat{x}^{i}=\hat{x}_{i}$ the $\kappa$-Minkowski defining commutator reads $\left[\hat{x}_{i}, \hat{x}_{0}\right]=\frac{i}{\kappa} \hat{x}_{i}$.

${ }^{2}$ It is easy to verify that the spatial generators are nilpotent, i.e., $\left[\left(\hat{x}_{i}\right)\right]^{3}=0$.
} 
noncommutative scenario with the usual commutative Minkowski spacetime. Since the noncommuting coordinates belong to a Lie algebra, plane waves will be elements of the corresponding Lie group and, accordingly, momenta will be coordinates on such group. Moreover, since $\kappa$-Minkowski coordinates are noncommuting objects, there will be inequivalent ordering prescription for defining a noncommutative plane wave. To fix the ideas, we will focus on the ordering convention where the time coordinate $\hat{x}^{0}$ appears to the right,

$$
g=e^{i k_{i} \hat{x}^{i}} e^{i k_{0} \hat{x}^{0}}
$$

The group element $g$ in the five-dimensional matrix representation reads

$$
G_{a}{ }^{b}=\left(\begin{array}{ccc}
\cosh \left(\frac{k_{0}}{\kappa}\right)+e^{\frac{k_{0}}{\kappa}} \frac{\mathbf{k}^{2}}{2 \kappa^{2}} & -\frac{1}{\kappa} \mathbf{k}^{T} & \sinh \left(\frac{k_{0}}{\kappa}\right)+e^{\frac{k_{0}}{\kappa}} \frac{\mathbf{k}^{2}}{2 \kappa^{2}} \\
-e^{\frac{k_{0}}{\kappa}} \frac{1}{\kappa} \mathbf{k} & \mathbb{I} & -e^{\frac{k_{0}}{\kappa}} \frac{1}{\kappa} \mathbf{k} \\
\sinh \left(\frac{k_{0}}{\kappa}\right)-e^{\frac{k_{0}}{\kappa}} \frac{\mathbf{k}^{2}}{2 \kappa^{2}} & \frac{1}{\kappa} \mathbf{k}^{T} & \cosh \left(\frac{k_{0}}{\kappa}\right)-e^{\frac{k_{0}}{\kappa}} \frac{\mathbf{k}^{2}}{2 \kappa^{2}}
\end{array}\right),
$$

where $\mathbb{I}$ denotes the $3 \times 3$ identity matrix and $\mathbf{k}^{T}=$ $\left(k_{1}, k_{2}, k_{3}\right)$. The choice of ordering (5) is quite natural in view of the Iwasawa decomposition defined above. In fact, the group element is nothing but the product of the two group elements $N=\exp \left(k_{i} \mathrm{n}_{i} / \kappa\right)$ and $A=\exp \left(k_{0} \mathrm{a} / \kappa\right)$ obtained by exponentiating the algebras $\mathfrak{n}$ and $\mathfrak{a}$, respectively. From the Iwasawa decomposition of the five-dimensional Lorentz group $S O(4,1)=S O(3,1) N A$, we can characterize the group generated by the $\kappa$-Minkowski Lie algebra as the quotient $S O(4,1) / S O(3,1) \sim N A$, also denoted in the literature as $A N(3)$ (for more details, see $[17,18]$ ). The real parameters $k_{0}$, $\mathbf{k}$ appearing in (5) are coordinates on the $A N(3)$ group momentum space known as "horospherical" coordinates [33].

In order to describe the manifold structure of the $A N(3)$ Lie group, we act with the generic element $g \in A N(3)$, in its $5 \times 5$ matrix representation $G$, on the spacelike vector $(0,0,0,0, \kappa)$ of the five-dimensional Minkowski (momentum) space. Writing the resulting vector in terms of global coordinates as $G \cdot(0,0,0,0, \kappa)=\left(P_{0}, P_{1}, P_{2}, P_{3}, P_{4}\right)$, one gets

$$
\begin{aligned}
& P_{0}=\kappa \sinh \left(\frac{k_{0}}{\kappa}\right)+e^{\frac{k_{0}}{\kappa}} \frac{\mathbf{k}^{2}}{2 \kappa} \\
& P_{i}=e^{\frac{k_{0}}{\kappa}} k_{i} \\
& P_{4}=\cosh \left(\frac{k_{0}}{\kappa}\right)-e^{\frac{k_{0}}{\kappa}} \frac{\mathbf{k}^{2}}{2 \kappa} .
\end{aligned}
$$

These coordinates satisfy the constraints

$$
-P_{0}^{2}+P^{i} P_{i}+P_{4}^{2}=\kappa^{2}, \quad P_{0}+P_{4}>0 .
$$

The former relation is nothing but the equation defining four-dimensional de Sitter space $d S_{4}$ in an embedding fivedimensional Minkowski space. The inequality $P_{0}+P_{4}>0$ restricts us to "half" of $d S_{4}$.

\section{A. The $\kappa$-Poincaré algebra}

As illustrated above, at the momentum space level, the noncommutativity of spacetime leads to momenta which belong to a non-Abelian Lie group rather than to a vector space as in ordinary relativistic kinematics. It is thus natural to expect that this basic structural shift will affect dramatically the ordinary notions of relativistic symmetries as described by the Poincare group. To understand how these structures are affected, let us consider the product of two "right-ordered" $A N(3)$ group elements $g=e^{i k_{i} \hat{x}^{i}} e^{i k_{0} \hat{x}^{0}}$ and $h=e^{i l_{i} \hat{x}^{i}} e^{i l_{0} \hat{x}^{0}}$. This can be written as

$$
g h=e^{i k_{i} \hat{x}^{i}} e^{i k_{0} \hat{x}^{0}} e^{i l_{i} \hat{x}^{i}} e^{i l_{0} \hat{x}^{0}}=e^{i\left(k_{i} \oplus l_{i}\right) \hat{x}^{i}} e^{i\left(k_{0} \oplus l_{0}\right) \hat{x}^{0}},
$$

where $k_{i} \oplus l_{i}=k_{i}+e^{-k_{0} / \kappa} l_{i}$ and $k_{0} \oplus l_{0}=k_{0}+l_{0}$. The addition law $k_{\mu} \oplus l_{\mu}=\left(k_{0} \oplus l_{0}, k_{i} \oplus l_{i}\right)$ is clearly nonAbelian, i.e., $k_{\mu} \oplus l_{\mu} \neq l_{\mu} \oplus k_{\mu}$, since the "momentum" Lie group is non-Abelian. The addition law for momenta reflects the composition of conserved quantum numbers associated to translation generators. In particular, the familiar addition of momenta can be seen as a consequence of the Leibiniz rule for the action of translation generators on multiparticle states. The non-Abelian composition of momenta thus reflects a deformed action of space translation generators. In the language of Hopf algebras [34], this can be expressed in terms of a nontrivial coproduct for the spatial translation generators $K_{i}$ which act diagonally on right-ordered plane waves

$$
\Delta K_{i}=K_{i} \otimes 1+e^{-K_{0} / \kappa} \otimes K_{i},
$$

while the time translation generator acts according to the usual Leibniz rule expressed by the trivial coproduct

$$
\Delta K_{0}=K_{0} \otimes 1+1 \otimes K_{0} .
$$

Notice that in the limit $\kappa \rightarrow \infty$, the coproduct (10) reduces to the trivial one. In a similar fashion, the group inversion is reflected in a nontrivial antipode for the generators

\footnotetext{
${ }^{3}$ The other half of $d S_{4}$, i.e., the one identified by the condition $P_{0}+P_{4}<0$, can be obtained by replacing the action of $G$ with $G \cdot \mathcal{N}$, where

$$
\mathcal{N}=\left(\begin{array}{ccc}
-1 & \mathbf{0}^{T} & 0 \\
\mathbf{0} & \mathbb{I} & \mathbf{0} \\
0 & \mathbf{0}^{T} & -1
\end{array}\right)
$$
}




$$
S\left(K_{0}\right)=-K_{0}, \quad S\left(K_{i}\right)=-e^{K_{0} / \kappa} K_{i},
$$

which determines the appropriate generalization of momentum subtraction operation $\ominus$ needed in order for the basic relation $k_{\mu} \oplus\left(\ominus k_{\mu}\right)=0$ to hold.

It is natural at this point to ask how these deformations of the action of translation generators affect the other generators of relativistic symmetries. In particular, whether the generators of rotations and boosts also exhibit nontrivial coproducts and antipodes, and if the deformations affect the ordinary structure of the commutators of the Poincare algebra. In general, both structures will be deformed. These nontrivial structures are mathematically described by a "quantum deformation" of the Poincaré algebra: the $\kappa$-Poincaré Hopf algebra introduced in [19]. It turns out that the Lorentz sector of the $\kappa$-Poincare algebra is characterized by trivial coproducts and antipodes for the generators of rotations, while those of the boost generators are deformed

$$
\begin{aligned}
\Delta M_{i} & =M_{i} \otimes 1+1 \otimes M_{i}, \quad S\left(M_{i}\right)=-M_{i}, \\
\Delta N_{i} & =N_{i} \otimes 1+e^{-K_{0} / \kappa} \otimes N_{i}+\frac{1}{\kappa} \varepsilon_{i j k} K^{j} \otimes M^{k}, \\
S\left(N_{i}\right) & =-e^{K_{0} / \kappa} N_{i}+\frac{1}{\kappa} \varepsilon_{i j k} e^{K_{0} / \kappa} K^{j} M^{k} .
\end{aligned}
$$

Notice that setting $K_{0}=K_{i}=0$, i.e., restricting to the Lorentz algebra, we recover a trivial Hopf algebra structure. The particular realization of the $\kappa$-Poincaré Hopf algebra in terms of the generators $\left\{K_{\mu}, M_{i}, N_{i}\right\}$, i.e., with translation generators associated to the parametrization of the $A N(3)$ group in terms of horospherical coordinates, is known in the literature as the bicrossproduct basis of the $\kappa$-Poincare algebra [16]. One of the characterizing features of the bicrossproduct basis is that, while translation generators behave as ordinary four vectors under rotations, the commutators between boosts and translation generators are deformed

$$
\begin{aligned}
{\left[K_{0}, N_{i}\right] } & =-i N_{i} \\
& =-i \delta_{i j}\left(\frac{\kappa}{2}\left(1-e^{-2 K_{0} / \kappa}\right)+\frac{1}{2 \kappa} K_{i} K^{i}\right)+\frac{i}{\kappa} K_{i} K_{j} .
\end{aligned}
$$

It can be shown [35] that the deformed commutator between boosts and spatial translation generators leads to finite boost transformations for which the modulus of the spatial momentum approaches the UV value of $\kappa$, rather than diverging, in the limit of infinite boost parameter. This behavior is typical of models based on nonlinear deformations of relativistic kinematics known as doubly special relativity [36-40], widely popular over the past fifteen years as effective models of Planck-scale kinematics incorporating the Planck energy, in our case identified with the UV deformation parameter $\kappa$, as an observer independent energy scale [41,42].

A rather important point to stress is that different choices of coordinates on the $A N(3)$ manifold will lead, in general, to different coproducts and antipodes for the associated translation generators. For example, the relations above can be used to derive the coproducts and antipodes for translation generators $P_{\mu}$ associated to the embedding coordinates defined in (7)

$\Delta\left(P_{0}\right)=P_{0} \otimes P_{+}+P_{+}^{-1} \otimes P_{0}+\frac{1}{\kappa} \sum_{i=1}^{3} P_{i} P_{+}^{-1} \otimes P_{i}$,

$\Delta\left(P_{i}\right)=P_{i} \otimes P_{+}+1 \otimes P_{i}$,

$\Delta\left(P_{4}\right)=P_{4} \otimes P_{+}-P_{+}^{-1} \otimes P_{0}-\frac{1}{\kappa} \sum_{i=1}^{3} P_{i} P_{+}^{-1} \otimes P_{i}$

$S\left(P_{0}\right)=-P_{0}+\frac{1}{\kappa} \mathbf{P}^{2} P_{+}^{-1}=\kappa P_{+}^{-1}-P_{4}$,

$S\left(P_{i}\right)=-P_{i} P_{+}^{-1}, \quad S\left(P_{4}\right)=P_{4}$,

where $P_{+} \equiv \frac{P_{0}+P_{4}}{\kappa}$. The corresponding coproducts and antipodes for rotations and boosts will be now given by

$\Delta\left(M_{i}\right)=M_{i} \otimes 1+1 \otimes M_{i}$

$\Delta\left(N_{i}\right)=N_{i} \otimes 1+P_{+}^{-1} \otimes N_{i}+\frac{\epsilon_{i j k}}{\kappa} P_{j} P_{+}^{-1} \otimes M_{k}$

$S\left(M_{i}\right)=-M_{i}$

$S\left(N_{i}\right)=-N_{i} P_{+}+\frac{\epsilon_{i j k}}{\kappa} P_{j} M_{k}$

These generators are known in the literature as the "classical" basis $[43,44]$ of the $\kappa$-Poincaré algebra since, unlike the bicrossproduct basis reviewed above, their commutators are just the ones of the ordinary Poincaré algebra. This also implies that the mass Casimir invariant naturally associated to the generators $P_{\mu}$ is just the ordinary one

$$
\mathcal{C}(P)=P_{\mu} P^{\mu} .
$$

In other words, in such a basis, the nontrivial features due to symmetry deformation manifest only in the "coalgebra" sector (i.e., in the coproducts and antipodes) leaving unmodified the familiar Lie algebra structure of relativistic symmetries.

Finally, let us notice that in terms of bicrossproduct generators the Casimir is no longer quadratic and takes the form

$$
\mathcal{C}(K)=\mathcal{C}_{\kappa}(K)\left(1+\frac{\mathcal{C}_{\kappa}(K)}{4 \kappa^{2}}\right)
$$


where $\mathcal{C}_{\kappa}(K)$ is the $\kappa$-deformed Casimir invariant naturally associated with the bicrossproduct basis [45]

$$
\mathcal{C}_{\kappa}(K)=\left(2 \kappa \sinh \left(\frac{K_{0}}{2 \kappa}\right)\right)^{2}-\mathbf{K}^{2} e^{K_{0} / \kappa}
$$

This Casimir determines a modification of the energymomentum dispersion relation governed by the UV scale $\kappa$ common to many models of departures from ordinary relativistic kinematics at the Planck scale. Possible signatures of such deformed dispersion relations in the highest energy astrophysical phenomena have been among the leading candidate scenarios for experimental manifestations of quantum gravity effects $[46,47]$.

\section{B. Noncommutative calculus}

As in ordinary field theory, one expects the Casimir invariant (20) to have a "coordinate space" counterpart in terms of a noncommutative wave operator. This will be written in terms of noncommutative differential operators complying with the nontrivial structure of the spacetime commutator (1) and of the symmetry generators. In this section, we introduce the differential calculus needed to define such operators (for further technical details, we refer the reader to $[48,49])$.

As it is well known in the literature [50], it is impossible to construct a four-dimensional set of noncommutative differentials which are also covariant under the action of $\kappa$-Poincaré generators. ${ }^{4}$ Rather, one has to resort to a five-dimensional set of noncommutative differentials $\left\{d \hat{x}_{0}, d \hat{x}_{1}, d \hat{x}_{2}, d \hat{x}_{3}, d \hat{x}_{4}\right\}$ with the following commutation relations with the $\kappa$-Minkowski coordinates:

$$
\begin{array}{rlrl}
{\left[\hat{x}_{0}, d \hat{x}_{0}\right]} & =\frac{i}{\kappa} d \hat{x}_{4}, & {\left[\hat{x}_{0}, d \hat{x}_{i}\right]=0,} & {\left[\hat{x}_{0}, d \hat{x}_{4}\right]=\frac{i}{\kappa} d \hat{x}_{0},} \\
{\left[\hat{x}_{i}, d \hat{x}_{0}\right]} & =\frac{i}{\kappa} d \hat{x}_{i}, & {\left[\hat{x}_{i}, d \hat{x}_{j}\right]=\delta_{i j} \frac{i}{\kappa}\left(d \hat{x}_{0}-d \hat{x}_{4}\right),} \\
{\left[\hat{x}_{i}, d \hat{x}_{4}\right]=\frac{i}{\kappa} d \hat{x}_{i} .} &
\end{array}
$$

It can be checked by taking the differential of both sides of (1) that these commutators are consistent with the noncommutative structure of spacetime and that all Jacobi identities involving differentials and noncommuting coordinates are satisfied. The Lorentz covariance of these commutators can be easily checked using the relations [16],

\footnotetext{
${ }^{4}$ For instance, the 4D differential calculus used in [51] and defined by the commutators

$$
\left[\hat{x}_{0}, d \hat{x}_{i}\right]=-\frac{i}{\kappa} d \hat{x}_{i}, \quad\left[\hat{x}_{0}, d \hat{x}_{0}\right]=0, \quad\left[\hat{x}_{i}, d \hat{x}_{\mu}\right]=0,
$$

is covariant with respect to the action of translations alone, but is not $\kappa$-Lorentz covariant.
}

$$
N_{i} \triangleright \hat{x}_{0}=i \hat{x}_{i}, \quad N_{i} \triangleright \hat{x}_{j}=i \delta_{i j} \hat{x}_{0},
$$

and extending the action to the algebra of differentials in a natural way as ${ }^{5}$

$$
\begin{aligned}
N_{i} \triangleright d \hat{x}_{\mu} & =d\left(N_{i} \triangleright \hat{x}_{\mu}\right), \\
N_{i} \triangleright\left(\hat{x}_{\mu} d \hat{x}_{\nu}\right) & =\left(N_{i}^{(1)} \triangleright \hat{x}_{\mu}\right)\left(d\left(N_{i}^{(2)} \triangleright \hat{x}_{\nu}\right)\right),
\end{aligned}
$$

where we have used Sweedler notation $\Delta(N)=N^{(1)} \otimes$ $N^{(2)}=\sum_{a} N^{(1) a} \otimes N^{(2) a}$ for the coproduct in (17).

A differential on the algebra of functions over $\kappa$ Minkowski spacetime can be defined as

$$
d=i d \hat{x}^{a} \hat{\partial}_{a},
$$

where the derivatives $\hat{\partial}_{a}$ are determined by requiring that the Leibniz rule for the differential is satisfied, as we now show. Working in the bicrossproduct basis, the explicit form of the $\hat{\partial}_{a}$ can be derived by first noting that, from the commutator $\left[\hat{x}_{\mu}, d \hat{x}_{a}\right]=\left(\hat{x}_{\mu}\right)^{b}{ }_{a} d \hat{x}_{b}$, follows the identity

$$
\hat{e}_{k} d \hat{x}^{a} \hat{e}_{\ominus k}=d \hat{x}^{b} G_{b}{ }^{a},
$$

where $G_{b}{ }^{a}$ is the matrix representation (6) of the rightordered plane wave $g=e^{i k_{i} \hat{x}^{i}} e^{i k_{0} \hat{x}^{0}} \equiv \hat{e}_{k}$. Imposing then the Leibniz rule for the differential $d$ on the product $\hat{e}_{k} \hat{e}_{q}$, we get

$$
\begin{aligned}
d\left(\hat{e}_{k} \hat{e}_{q}\right) & =\left(d \hat{e}_{k}\right) \hat{e}_{q}+\hat{e}_{k}\left(d \hat{e}_{q}\right) \\
& =\left(i d \hat{x}^{a} \hat{\partial}_{a} \hat{e}_{k}\right) \hat{e}_{q}+\hat{e}_{k}\left(i d \hat{x}^{a} \hat{\partial}_{a} \hat{e}_{q}\right) \\
& =i\left[\left(d \hat{x}^{a} \hat{\partial}_{a} \hat{e}_{k}\right) \hat{e}_{q}+\hat{e}_{k}\left(d \hat{x}^{a} \hat{e}_{\ominus k} \hat{e}_{k} \hat{\partial}_{a} \hat{e}_{q}\right)\right] \\
& =i d \hat{x}^{a}\left[\left(\hat{\partial}_{a} \hat{e}_{k}\right) \hat{e}_{q}+\left(G_{a}^{b} \hat{e}_{k}\right)\left(\hat{\partial}_{b} \hat{e}_{q}\right)\right],
\end{aligned}
$$

where in the second term of the third equality, we have introduced $\hat{e}_{\ominus k} \hat{e}_{k}=1$ and in the last equality, we used the relation (27). Accordingly, looking at the first and the last terms of (28), we find that, in order for the differential $d=i d \hat{x}^{a} \hat{\partial}_{a}$ to satisfy the Leibniz rule, the derivatives must have coproducts

$$
\Delta\left(\hat{\partial}_{a}\right)=\hat{\partial}_{a} \otimes 1+G_{a}^{b} \otimes \hat{\partial}_{b} .
$$

It turns out that these coproducts reproduce the ones for the classical basis generators $P_{a}$ in (15), with the coproduct of the operator $\hat{\partial}_{4}$ corresponding to the coproduct of $\left(\kappa-P_{4}\right)$. Therefore, we can identify noncommutative derivatives associated with the five-dimensional covariant calculus with translation generators of the classical basis. The action of the derivatives on right-ordered plane waves is then

\footnotetext{
${ }^{5} \mathrm{We}$ also assume that the differential $d \hat{x}_{4}$ is $\kappa$-Poincaré invariant $\mathcal{P}_{\kappa} \triangleright d \hat{x}_{4}=0$, where $\mathcal{P}_{\kappa}$ is a generic element of the $\kappa$-Poincaré algebra.
} 


$$
\hat{\partial}_{\mu} \hat{e}_{k}=P_{\mu}(k) \hat{e}_{k}, \quad \hat{\partial}_{4} \hat{e}_{k}=\left(\kappa-P_{4}(k)\right) \hat{e}_{k},
$$

where the explicit form of the classical basis momenta $P_{a}(k)$ in terms of the bicrossproduct momenta is given by (7). One also defines conjugate operators $\hat{\partial}_{\mu}^{\dagger}$ whose action on plane waves is given by ${ }^{6}$

$$
\hat{\partial}_{\mu}^{\dagger} \hat{e}_{k} \equiv\left(\hat{\partial}_{\mu} \hat{e}_{k}^{\dagger}\right)^{\dagger}=S(P(k))_{\mu} \hat{e}_{k}, \quad \hat{\partial}_{4}^{\dagger}=\hat{\partial}_{4},
$$

reflecting the fact that $P(S(k))_{a}=S(P(k))_{a}$.

From the action of the derivatives on $\hat{e}_{k}$, one can straightforwardly derive the action of the operators $\hat{\partial}_{a}$ on the generic function of noncommuting coordinates $\hat{f}(\hat{x})$. This can be done by resorting to the following Fourier expansion [32,52-54] in terms of right-ordered noncommutative plane waves $\hat{e}_{k}$ :

$$
\hat{f}(\hat{x})=\int d \mu(k) \tilde{f}_{r}(k) \hat{e}_{k}(\hat{x}),
$$

where the integration measure $d \mu(k)$ is the Haar measure ${ }^{7}$ on $A N(3)$,

$$
d \mu(k)=\frac{e^{3 k_{0} / \kappa}}{(2 \pi)^{4}} d k_{0} d \mathbf{k}
$$

which can be also expressed in terms of the ordinary Lebesgue measure on the five-dimensional embedding space $d^{5} P$ as

$$
d \mu(P)=\kappa \frac{\delta\left(P_{a} P^{a}-\kappa^{2}\right) \theta\left(P_{0}+P_{4}\right)}{(2 \pi)^{4}} d^{5} P,
$$

and the subscript of $\tilde{f}_{r}(k)$ denotes that the Fourier transform is defined in terms of right-ordered noncommutative plane waves.

\section{Weyl maps and $\star$ product}

The Weyl map is a useful tool first introduced in quantum mechanics to map classical observables (commuting functions on phase space) to quantum observables (functions of noncommuting operators). Because of ordering ambiguities on the noncommutative side, Weyl maps are obviously not unique. In our context, a Weyl map will map a function on the commutative Minkowski space to a

\footnotetext{
${ }^{6}$ Here, we have used the fact that the Hermitian conjugate of a plane wave involves the antipode map $S(p)$ on its momentum $\hat{e}_{k}^{\dagger}=\hat{e}_{S(k)} \equiv \hat{e}_{\ominus k}$.

${ }^{7}$ It is a left invariant measure $d \mu(p k)=d \mu(k)$, and it is worth noticing that in horospherical coordinates, it is just the diffeomorphism invariant measure on $d S_{4}$ corresponding to the cosmological metric $-d k_{0}^{2}+e^{2 k_{0} / \kappa} d k_{i}^{2}$.
}

(suitably ordered) function on the noncommutative $\kappa$ Minkowski space.

Let us focus on plane waves. As we will see, in our context, the ordering ambiguity will reflect different choices of bases for the $\kappa$-deformed translation generators. We define the "right-ordered" Weyl map $\Omega_{r}$ as

$$
\Omega_{r}\left(e^{i k x}\right)=e^{i k_{i} \hat{x}^{i}} e^{i k_{0} \hat{x}^{0}}=\hat{e}_{k}, \quad \Omega_{r}^{-1}\left(\hat{e}_{k}\right)=e^{i k x}
$$

i.e., $\Omega_{r}$ an ordinary plane wave is mapped to an $A N(3)$ group element written in the decomposition (5) in which the non-Abelian generator $\hat{x}^{0}$ is always to the right. One can associate a commutative function $f_{r}(x)$ to $\hat{f}(\hat{x})$ via this Weyl map using the Fourier expansion (32)

$$
\begin{aligned}
f_{r}(x) & =\Omega_{r}^{-1}(\hat{f}(\hat{x}))=\int d \mu(k) \tilde{f}_{r}(k) \Omega_{r}^{-1}\left(\hat{e}_{k}(\hat{x})\right) \\
& =\int d \mu(k) \tilde{f}_{r}(k) e^{i k x} .
\end{aligned}
$$

Among the possible Weyl maps to functions on $\kappa$ Minkowski, a preferred choice, which we denote $\Omega_{c}$, is given by the map leading to noncommutative plane waves on which the derivatives $\hat{\partial}_{\mu}$ of the noncommutative differential calculus have a "classical" action; i.e.,

$$
\hat{\partial}_{\mu} \triangleright \Omega_{c}\left(e^{i p x}\right)=\Omega_{c}\left(-i \partial_{\mu} e^{i p x}\right)=p_{\mu} \Omega_{c}\left(e^{i p x}\right) .
$$

The Weyl map $\Omega_{c}$ is related to the classical basis coordinates $P_{a}$ and, as it can be easily checked confronting the actions (30) and (37), it has the following action on plane waves:

$$
\Omega_{c}\left(e^{i P x}\right)=\hat{e}_{k(P)}, \quad \Omega_{c}^{-1}\left(\hat{e}_{k(P)}\right)=e^{i P x},
$$

that is, $\Omega_{c}$ maps a commutative plane wave labeled by $P$ to a right-ordered noncommutative plane wave whose four-momentum is $k_{\mu}(P)$, where $k(P)$ is the inverse transformation of (7). Therefore, following (32), a noncommutative function $\hat{f}(\hat{x})$ can be expressed as

$$
\hat{f}(\hat{x})=\int d \mu(P) \tilde{f}_{c}(P) \Omega_{c}\left(e^{i P x}\right),
$$

where $\tilde{f}_{c}(P)=\tilde{f}_{r}(k(P))$, and the commutative function $f_{c}(x)$ associated to $\hat{f}(\hat{x})$ through the inverse classical basis Weyl map is given by

$$
f_{c}(x)=\Omega_{c}^{-1}(\hat{f}(\hat{x}))=\int d \mu(P) \tilde{f}_{c}(P) e^{i P x} .
$$

Using such a map, we can finally introduce a suitable notion of integration on $\kappa$-Minkowski space as follows: 


$$
\widehat{\int} \hat{f} \equiv \int d^{4} x \Omega_{c}^{-1}(\hat{f}(\hat{x}))=\int d^{4} x f_{c}(x)
$$

On the space of commutative functions obtained via the action of $\Omega_{c}^{-1}$, the noncommutativity of $\kappa$-Minkowski space is reflected in a nontrivial $\star$ product which replaces the ordinary commutative pointwise product. The star product associated to the Weyl map $\Omega_{c}$ is defined by the relation

$\hat{f}(\hat{x}) \hat{g}(\hat{x})=\Omega_{c}\left(f_{c}(x)\right) \Omega_{c}\left(g_{c}(x)\right)=\Omega_{c}\left(f_{c}(x) \star g_{c}(x)\right)$.

It can be shown [32] that the explicit formula for products of the form $f_{c}^{\dagger} \star g_{c}=\Omega_{c}^{-1}\left(\hat{f}^{\dagger} \hat{g}\right)$ has the rather simple expression

$$
f_{c}^{\dagger}(x) \star g_{c}(x)=f_{c}^{*}(x) \sqrt{1+\square / \kappa^{2}} g_{c}(x),
$$

where with $f_{c}^{\dagger}$ we denote the $\kappa$-Minkowski Hermitian conjugation involving the antipode, e.g., $\left(e^{i P x}\right)^{\dagger}=e^{i S(P) x}$, while $f_{c}^{*}$ is just the standard complex conjugation. The $\star$ product (43) can be used to define the Fourier transform

$\tilde{f}_{c}(P)=\widehat{\int}\left[\Omega_{c}\left(e^{i P x}\right)\right]^{\dagger} \hat{f}(\hat{x})=\int d^{4} x\left(e^{i P x}\right)^{\dagger} \star f_{c}(x)$,

which, taking into account the explicit form of the integration measure and the $\star$ product, leads to the two fundamental relations

$$
\tilde{f}_{c}(P)=\frac{\left|P_{4}\right|}{\kappa} \int d^{4} x e^{-i P x} f_{c}(x)
$$

and

$$
f_{c}(x)=\int \frac{d^{4} P \theta\left(P_{0}+P_{4}\right)}{(2 \pi)^{4}\left|P_{4}\right| / \kappa} \tilde{f}_{c}(P) e^{i P x},
$$

where $P_{4}= \pm \sqrt{\kappa^{2}-P^{2}}$ and $P^{2}=-P_{0}^{2}+\mathbf{P}^{2}$. It is worth noticing that the operator $\sqrt{1+\square / \kappa^{2}}$, coming from the $\star$ product, in momentum space is nothing but $\left|P_{4}\right| / \kappa$, i.e., the same term appearing in the denominator of the integration measure; this will lead to important simplifications in what follows.

We conclude this introductory section with some remarks on Lorentz invariance. As first noted in [52] and successively elaborated in [55], the momentum space suffers from a subtle form of Lorentz symmetry breaking. Namely, for any negative energy mode, the allowed range of rapidities is bounded above. As we discussed in the previous sections, the bicrossproduct coordinates cover only half of de Sitter space identified by the condition $\kappa P_{+}(k) \equiv P_{0}(k)+P_{4}(k)>0$, so that the momentum space is not the whole de Sitter space $d S_{4}$. In the classical basis, this restriction explicitly breaks Lorentz invariance since it is not preserved by boosts (remember that $P_{0}$ transform as the 0 th component of a Lorentz vector while $P_{4}$ is a Lorentz scalar). Indeed, it takes a boost with finite rapidity to bring a point out of the region $P_{0}+P_{4}>0$.

A way to circumvent this problem is to take as momentum space the full de Sitter space quotiented by reflections $P_{a} \rightarrow-P_{a}$. In fact, by reflections, the sector $P_{0}+P_{4}>0$ is sent to its complement. This space is called the elliptic de Sitter space $d S_{4} / \mathbb{Z}_{2}$. Accordingly, one can change the defining condition from $P_{0}+P_{4}>0$ to $P_{4}>0$, which is clearly Lorentz invariant, by considering, instead of the sector identified by the conditions $P_{0}+P_{4}>0$ and $P_{4}<0$, its image under reflection, i.e., the sector with $P_{0}+P_{4}<0$ and $P_{4}>0$. The Fourier transform $\tilde{f}_{c}(P)$, defined so far only in the region $P_{+}>0$, is now defined on the whole de Sitter momentum space, which is even under the $\mathbb{Z}_{2}$ identification $\tilde{f}_{c}\left(P_{a}\right)=\tilde{f}_{c}\left(-P_{a}\right)$. This suggest that, in the classical basis, the Lorentz invariant measure on $d S_{4} / \mathbb{Z}_{2}$ will be

$$
d \mu(P)=2 \kappa \frac{\delta\left(P_{a} P^{a}-\kappa^{2}\right) \theta\left(P_{4}\right)}{(2 \pi)^{4}} d^{5} P .
$$

Solving the delta function with respect to $P_{4}$, we have that a noncommutative function $\hat{f}(\hat{x})$ can be Fourier expanded as

$$
\hat{f}(\hat{x})=\int \frac{d^{4} P \theta\left(\kappa^{2}-P^{2}\right)}{(2 \pi)^{4}\left|P_{4}\right| / \kappa} \tilde{f}_{c}(P) \Omega_{c}\left(e^{i P x}\right),
$$

where the Heaviside step function ensures that $P_{4}=$ $\sqrt{\kappa^{2}-P^{2}} \in \mathbb{R}$.

\section{FREE $\kappa$-DEFORMED QUANTUM FIELDS: THE FEYNMAN PROPAGATOR}

\section{A. The $\boldsymbol{\kappa}$-deformed free field partition function}

We now move to the study of $\kappa$-deformed quantum fields. The $\kappa$-Poincaré invariant action of a free massive complex scalar field is given by

$$
S_{\text {free }}\left[\hat{\phi}, \hat{\phi}^{\dagger}\right]=\widehat{\int}\left[\left(\hat{\partial}_{\mu} \hat{\phi}\right)^{\dagger}\left(\hat{\partial}^{\mu} \hat{\phi}\right)+m^{2} \hat{\phi}^{\dagger} \hat{\phi}\right] .
$$

The derivatives $\hat{\partial}_{\mu}$ are those of the 5D bicovariant and $\kappa$ Poincaré covariant differential calculus illustrated in Sec. II. From the action (49), making use of the coproduct properties of the $\hat{\partial}_{\mu}$ 's [recall that $\Delta\left(\hat{\partial}_{\mu}\right)=\Delta\left(P_{\mu}\right)$ ], one obtains the following equation of motion:

$$
\left(\hat{\partial}_{\mu} \hat{\partial}^{\mu}+m^{2}\right) \hat{\phi}(\hat{x})=0,
$$

and an identical one for $\hat{\phi}^{\dagger}(\hat{x})$ thanks to the property $\left(\hat{\partial}_{\mu} \hat{\partial}^{\mu}\right)^{\dagger}=\hat{\partial}_{\mu} \hat{\partial}^{\mu}$, which reflects the fact that, in the classical basis, the antipodes satisfy the relation $S(P)_{\mu} S(P)^{\mu}=$ $P_{\mu} P^{\mu}$. Considering the Fourier expansion (48), the free action (49) can be expressed in momentum space as 


$$
S_{\text {free }}\left[\tilde{\phi}, \tilde{\phi}^{*}\right]=\int \frac{d \bar{\mu}(p)}{(2 \pi)^{4}} \tilde{\phi}^{*}(p)\left(p_{\mu} p^{\mu}+m^{2}\right) \tilde{\phi}(p),
$$

where the measure is $d \bar{\mu}(p)=d^{4} p \theta\left(\kappa^{2}-p^{2}\right) \kappa /\left|p_{4}\right|$, and we denoted the classical basis momenta with $p_{a}$ and the commutative functions $\tilde{\phi}_{c}(p)$ simply with $\tilde{\phi}(p)$. In deriving this last expression, we have also used the relation (37) for the action of derivatives on plane waves and the following relation for integration on $\kappa$-Minkowski:

$$
\begin{aligned}
\int_{\hat{x}} & \left.\Omega_{c}\left(e^{i p x}\right)\right]^{\dagger} \Omega_{c}\left(e^{i q x}\right) \\
& =\int d^{4} x\left(e^{i p_{\mu} x^{\mu}}\right)^{*} \sqrt{1+\square / \kappa^{2}}\left(e^{i q_{\mu} x^{\mu}}\right) \\
& =(2 \pi)^{4} \frac{\left|p_{4}\right|}{\kappa} \delta\left(p_{0}-q_{0}\right) \delta(\mathbf{p}-\mathbf{q}) .
\end{aligned}
$$

Looking at the expression of the action (51), the simplifications introduced by working with the classical basis of the $\kappa$-Poincaré algebra become evident. Indeed, as a result of the fact that in the classical basis the algebraic sector is undeformed, the Casimir $\mathcal{C}=p_{\mu} p^{\mu}$ appearing in (51) is the standard one. Therefore, the momentum space free action differs from the ordinary one only for the integration measure $d \bar{\mu}(p)$.

The action in momentum space (51) can be used to write down the partition function of the theory. A partition function obtained from a momentum space action of a $\kappa$-deformed field was first used in [29]. However, at that time, a full understanding of the momentum space related to the $\kappa$-Poincare Hopf algebra had not yet been reached. Specifically, the fact that the space of momenta is the fourdimensional elliptic de Sitter space was not taken into account, and consequently, the exact form of the momentum space integration measure was not given explicitly in the analysis of [29]. A more recent use of the $\kappa$-deformed partition function, which implemented the nontrivial geometric features of the momentum space, has appeared in [28]. This work presented a field theoretic approach to the study of the potential between two static point sources in a noncommutative space. The partition function adopted in [28] can be straightforwardly generalized to the complex scalar field case as

$$
\bar{Z}\left[J, J^{\dagger}\right]=\int \mathcal{D}[\phi] \mathcal{D}\left[\phi^{\dagger}\right] e^{i S_{\text {free }}\left[\hat{\phi}, \hat{\phi}^{\dagger}\right]+i \int\left[\hat{\phi}^{\dagger} \hat{J}+\hat{J}^{\dagger} \hat{\phi}\right]},
$$

where the action $S_{\text {free }}\left[\hat{\phi}, \hat{\phi}^{\dagger}\right]$ is the $\kappa$-Poincaré invariant action (49). We focus on the normalized partition function

$$
Z\left[J, J^{\dagger}\right]=\frac{\bar{Z}\left[J, J^{\dagger}\right]}{\bar{Z}[0,0]} .
$$

In order to bring $Z\left[J, J^{\dagger}\right]$ into a well-suited expression for the manipulation needed to extract the Feynman propagator, we rewrite the partition function in momentum space. Indeed, since the momentum space is a commutative space, here it is possible to handle the functional calculus (which we will illustrate below) unambiguously. Making use of (48), (51), and (52), one obtains from (53)

$$
Z\left[\tilde{J}, \tilde{J}^{*}\right]=\frac{1}{\bar{Z}[0,0]} \int \mathcal{D}[\tilde{\phi}] \mathcal{D}\left[\tilde{\phi}^{*}\right] e^{i \int \frac{d \bar{\mu}(p)}{(2 \pi)^{4}}\left[\tilde{\phi}^{*}(p)\left(p_{\mu} p^{\mu}+m^{2}\right) \tilde{\phi}(p)+\tilde{\phi}^{*}(p) \tilde{J}(p)+\tilde{J}^{*}(p) \tilde{\phi}(p)\right]}
$$

The functional integration can now be carried out as an ordinary Gaussian integral and, after simple manipulations, one finds that

$$
Z\left[\tilde{J}, \tilde{J}^{*}\right]=\exp \left(i \int \frac{d \bar{\mu}(p)}{(2 \pi)^{4}} \frac{\tilde{J}^{*}(p) \tilde{J}(p)}{-p^{2}-m^{2}+i \varepsilon}\right)
$$

In this last expression, we introduced the usual shift $m^{2} \rightarrow m^{2}-i \varepsilon$ to render the integral well defined.

In order to derive the Feynman propagator from the partition function $Z\left[\tilde{J}, \tilde{J}^{*}\right]$, we need an appropriate generalization of the functional derivatives to the deformed setting. In particular, one has to take into account the $\kappa$-deformed coproduct structure of the translation generators in (15), which leads to the following non-Abelian addition laws for momenta:

$$
\begin{aligned}
& (p \oplus q)_{0}=p_{0} q_{+}+\frac{q_{0}}{p_{+}}+\frac{1}{\kappa} \frac{p_{i} q^{i}}{p_{+}} \\
& (p \oplus q)_{i}=p_{i} q_{+}+q_{i} .
\end{aligned}
$$

This issue was first faced in [29] where however, as recalled above, the explicit form of the momentum space integration measure was not taken into account. Nonetheless, for an explicit definition of the functional derivatives such information is needed. Indeed, an important ingredient in the construction of functional calculus is a notion of delta function on the space of momenta. We will consider a delta function compatible with the nontrivial momentum space measure $d \bar{\mu}(p)$ [56-58], i.e., such that

$$
\int d \bar{\mu}(q) \delta(p, q) f(q)=f(p) .
$$


It can be easily checked that such delta function is given by

$$
\delta(p, q)=\delta((\ominus p) \oplus q)=\frac{\left|p_{4}\right|}{\kappa} \delta(p-q),
$$

and thus, it is proportional to an ordinary delta function $\delta(p-q)$, and, in particular, it is symmetric under the exchange of momenta in the argument $\delta((\ominus p) \oplus q)=$ $\delta((\ominus q) \oplus p)$. Let us mention that the other possible choice of delta function $\delta(p \oplus(\ominus q))$ would have been less natural since it carries and additional multiplicative factor, indeed from

$\delta(p \oplus(\ominus q))=\left|p_{+}\right|^{3} \frac{\left|p_{4}\right|}{\kappa} \delta(p-q)=\left|p_{+}\right|^{3} \delta((\ominus p) \oplus q)$,

it is easily seen that

$$
\int d \bar{\mu}(q) \delta(p \oplus(\ominus q)) f(q)=\left|p_{+}\right|^{3} f(p) .
$$

Notice that the two delta functions $\delta((\ominus p) \oplus q)$ and $\delta(p \oplus(\ominus q))$ are related by the antipode transformation $(p, q) \rightarrow(\ominus p, \ominus q)$, and thus, the appearance of the factor $\left|p_{+}\right|^{3}$ is related to the Jacobian of the antipode map $\left|J\left\{\frac{\partial \ominus p}{\partial p}\right\}\right|=\left|p_{+}^{-1}\right|^{3}$.

With the choice of the delta function (60), we can now proceed as in [29], though specializing the discussion to a complex field, and define the following $\kappa$-deformed functional derivatives:

$$
\begin{aligned}
\frac{\delta Z\left[\tilde{J}, \tilde{J}^{*}\right]}{\delta \tilde{J}(q)}= & \lim _{\epsilon \rightarrow 0} \frac{1}{\epsilon}\left\{Z\left[\tilde{J}(p)+\epsilon \delta((\ominus p) \oplus q), \tilde{J}^{*}(p)\right]\right. \\
& \left.-Z\left[\tilde{J}, \tilde{J}^{*}\right]\right\}, \\
\frac{\delta Z\left[\tilde{J}, \tilde{J}^{*}\right]}{\delta \tilde{J}^{*}(q)}= & \lim _{\epsilon \rightarrow 0} \frac{1}{\epsilon}\left\{Z\left[\tilde{J}(p), \tilde{J}^{*}(p)+\epsilon \delta((\ominus p) \oplus(\ominus q))\right]\right. \\
& \left.-Z\left[\tilde{J}, \tilde{J}^{*}\right]\right\},
\end{aligned}
$$

which clearly reduce to the ordinary definitions in the limit $\kappa \rightarrow \infty$, given that $\ominus p \rightarrow-p$ and $p \oplus q \rightarrow p+q$. These will be employed in the next section to obtain the Feynman propagator on $\kappa$-Minkowski noncommutative space.

\section{B. The Feynman propagator}

Following [29], we define the $\kappa$-deformed Feynman propagator in terms of the functional derivative of the partition function (56) with respect to incoming and outgoing source functions,

$i \tilde{\Delta}_{F}^{\kappa}(p, q)=\left.\left(i \frac{\delta}{\delta \tilde{J}^{*}(p)}\right)\left(-i \frac{\delta}{\delta \tilde{J}(q)}\right) Z\left[\tilde{J}, \tilde{J}^{*}\right]\right|_{\tilde{J}_{,} \tilde{J}^{*}=0}$.
Taking into account the relations (63), one obtains

$$
\begin{aligned}
i \tilde{\Delta}_{F}^{\kappa}(p, q) & =i(2 \pi)^{4} \frac{\delta((\ominus q) \oplus(\ominus p))}{-q^{2}-m^{2}+i \varepsilon} \\
& =i(2 \pi)^{4} \frac{\left|q_{4}\right|}{\kappa} \frac{\delta(q-S(p))}{-q^{2}-m^{2}+i \varepsilon}
\end{aligned}
$$

where in the last equality, we have expanded the delta function as in (60). Through the inverse Fourier transform (48), it is then possible to obtain the free scalar Feynman propagator on $\kappa$-Minkowski noncommutative space

$$
i \hat{\Delta}_{F}^{\kappa}(\hat{x}, \hat{y})=i \int \frac{d \bar{\mu}(p)}{(2 \pi)^{4}} \frac{\Omega_{c}\left(e^{i p x}\right)\left[\Omega_{c}\left(e^{i p y}\right)\right]^{\dagger}}{-p^{2}-m^{2}+i \varepsilon} .
$$

A point that deserves to be stressed is that $i \hat{\Delta}_{F}^{\kappa}(\hat{x}, \hat{y})$ is not symmetric under an exchange of its arguments. This property of the Feynman propagator (66) originates from the fact that, in the $\kappa$-deformed setting, the Hermitian conjugate of a plane wave involves the antipode map $S(p)$ on its momentum. Such spacetime asymmetry of $i \hat{\Delta}_{F}^{k}(\hat{x}, \hat{y})$, as we will see, although it may seem puzzling, does not lead to an actual physical asymmetry of the $\kappa$-Minkowski field propagation. Nonetheless, the combination of noncommutative plane waves appearing in (66), which is the cause of this concern, makes sure that the Feynman propagator is a Green's function of the $\kappa$-Klein Gordon equation (50), as we now show. As a first step, we define the noncommutative delta function $\hat{\delta}(\hat{x}, \hat{y})$ using the $\kappa$-Minkowski Fourier transform and antitransform (44) and (48)

$$
\begin{aligned}
\hat{f}(\hat{x}) & =\int \frac{d \bar{\mu}(p)}{(2 \pi)^{4}} \tilde{f}(p) \Omega_{c}\left(e^{i p x}\right) \\
& =\int \frac{d \bar{\mu}(p)}{(2 \pi)^{4}}\left(\int_{\hat{y}}\left[\Omega_{c}\left(e^{i p y}\right)\right]^{\dagger} \hat{f}(\hat{y})\right) \Omega_{c}\left(e^{i p x}\right) .
\end{aligned}
$$

By requiring

$$
\hat{f}(\hat{x})=\widehat{\int}_{\hat{y}} \hat{\delta}(\hat{x}, \hat{y}) \hat{f}(\hat{y}),
$$

we are led to define the noncommutative $\delta$-function ${ }^{8}$

$$
\hat{\delta}(\hat{x}, \hat{y})=\int \frac{d \bar{\mu}(p)}{(2 \pi)^{4}} \Omega_{c}\left(e^{i p x}\right)\left[\Omega_{c}\left(e^{i p y}\right)\right]^{\dagger} .
$$

Applying the $\kappa$-Klein-Gordon operator to the Feynman propagator (66) and taking into account the expression of the delta function (69), we thus get

\footnotetext{
${ }^{8}$ The noncommutative delta function $\hat{\delta}(\hat{x}, \hat{y})$ also satisfies
}

$$
\widehat{\int_{\hat{x}, \hat{y}}} \hat{\delta}(\hat{x}, \hat{y})=1
$$




$$
\left(\hat{\partial}_{\mu} \hat{\partial}^{\mu}+m^{2}\right) \hat{\Delta}_{F}^{\kappa}(\hat{x}, \hat{y})=-\hat{\delta}(\hat{x}, \hat{y})
$$

which shows that $\hat{\Delta}_{F}^{\kappa}(\hat{x}, \hat{y})$ is a (noncommutative) Green's function for this operator.

We now get back to the issue of the spacetime asymmetry of the $\kappa$-deformed propagator raised above and study how $\hat{\Delta}_{F}^{\kappa}(\hat{x}, \hat{y})$ propagates the field in the presence of a perturbation generated by an external source $\hat{J}(\hat{x})$. This exercise will also provide significant insight on the physical properties of the $\kappa$-Minkowski Feynman propagator. Given the $\kappa$-Klein-Gordon equation in the presence of a source,

$$
\left(\hat{\partial}_{\mu} \hat{\partial}^{\mu}+m^{2}\right) \hat{\phi}(\hat{x})=\hat{J}(\hat{x}),
$$

using the Eqs. (68) and (70), we can write down the following solution:

$$
\hat{\phi}(\hat{x})=-\widehat{\int}_{\hat{y}} \hat{\Delta}_{F}^{\kappa}(\hat{x}, \hat{y}) \hat{J}(\hat{y})
$$

In contrast with the standard commutative case, the $\kappa$-Minkowski integral (72) involves the product of two noncommutative functions and requires some additional care. As recalled in the previous section, such an integral can be defined via the ordinary Lebesgue integral introducing a noncommutaive $\star$ multiplication between the fields. Indeed, writing the source $\hat{J}(\hat{y})$ as a Fourier integral,

$$
\hat{J}(\hat{y})=\int \frac{d \bar{\mu}(p)}{(2 \pi)^{4}} \tilde{J}(p) \Omega_{c}\left(e^{i p y}\right),
$$

we see that (72) involves an integral of the form,

$$
\widehat{\int_{\hat{y}}}\left[\Omega_{c}\left(e^{i p y}\right)\right]^{\dagger} \Omega_{c}\left(e^{i q y}\right) .
$$

This is just the integral in (52) which, making use of the inverse Weyl map $\Omega_{c}^{-1}$ and its associated star product, can be expressed as

$$
\begin{aligned}
\widehat{\int}_{\hat{y}}\left[\Omega_{c}\left(e^{i p y}\right)\right]^{\dagger} \Omega_{c}\left(e^{i q y}\right) & =\int d^{4} y\left(e^{i S(p)_{\mu} y^{\mu}}\right) \star\left(e^{i q_{\mu} y^{\mu}}\right) \\
& =\int d^{4} y e^{-i p_{\mu} y^{\mu}} \sqrt{1+\square / \kappa^{2}} e^{i q_{\mu} y^{\mu}} .
\end{aligned}
$$

We have therefore that the propagation law (72) can be expressed in terms of commutative fields as

$$
\phi(x)=-\int d^{4} y \Delta_{F}^{\kappa}(x-y) \sqrt{1+\square / \kappa^{2}} J(y),
$$

where $\phi(x)=\Omega_{c}^{-1}(\hat{\phi}(\hat{x})), \quad J(y)=\Omega_{c}^{-1}(\hat{J}(\hat{y}))$, and we defined the $\kappa$-deformed Feynman propagator on commutative Minkowski space

$$
i \Delta_{F}^{\kappa}(x-y)=i \int \frac{d \bar{\mu}(p)}{(2 \pi)^{4}} \frac{e^{i p(x-y)}}{-p^{2}-m^{2}+i \varepsilon} .
$$

From (76), we see that the spacetime asymmetry of the $\kappa$-Minkowski Feynman propagator (66) does not affect the actual propagation of the field. Indeed, such asymmetry is canceled by the star product of (75), leading to a field propagation governed by the spacetime symmetric $\kappa$-deformed Feynman propagator (77).

Let us also notice that in the propagation law (76) we can make the star product term $\sqrt{1+\square / \kappa^{2}}$ act either on the source $J(y)$ or, equivalently, ${ }^{9}$ on the $\kappa$-deformed propagator $\Delta_{F}^{\kappa}(x-y)$.

Acting on $J(y)$ and noticing that in momentum space the term $\sqrt{1+\square / \kappa^{2}}$ is equal to $\left|p_{4}\right| / \kappa$, so that it cancels the same factor in the nontrivial integration measure $d \bar{\mu}(p)$, one obtains

$$
\phi(x)=-\int d^{4} y \Delta_{F}^{\kappa}(x-y) J_{c l}(y),
$$

where $J_{c l}(y)$ is a classical source

$$
J_{c l}(y)=\int \frac{d^{4} p}{(2 \pi)^{4}} \tilde{J}(p) e^{i p y},
$$

i.e., just an ordinary commutative function which, in particular, can describe a sharply localized source (e.g., a Dirac delta function).

Acting instead with the star product term $\sqrt{1+\square / \kappa^{2}}$ in (76) on $\Delta_{F}^{\kappa}(x-y)$, one gets

$$
\phi(x)=-\int d^{4} y \Delta_{F}(x-y) J(y),
$$

where $i \Delta_{F}(x-y)$ is the undeformed free scalar Feynman propagator

$$
i \Delta_{F}(x-y)=i \int \frac{d^{4} p}{(2 \pi)^{4}} \frac{e^{i p(x-y)}}{-p^{2}-m^{2}+i \varepsilon},
$$

and where, in this case, the source function $J(y)$ can not describe a pointlike source due to the presence of the $\kappa$-deformed integration measure $d \bar{\mu}(p)=d^{4} p \theta\left(\kappa^{2}-p^{2}\right) \kappa /$ $\left|p_{4}\right|$ in its Fourier expansion (73). The source function $J(y)$

\footnotetext{
${ }^{9}$ Given the propagation law $\phi(x)=-\int d^{4} y \Delta_{F}^{\kappa}(x-y) \times$ $\sqrt{1+\square / \kappa^{2}} J(y)$, we can take the formal series expansion in powers of the d'Alembertian for the star product term $\phi(x)=$ $-\int d^{4} y \sum_{n=0}^{\infty} a_{n} \Delta_{F}^{\kappa}(x-y) \square^{n} J(y)$ which, after integrating by parts, becomes $\phi(x)=-\int d^{4} y \sum_{n=0}^{\infty} a_{n} \square^{n} \Delta_{F}^{\kappa}(x-y) J(y)$.
} 


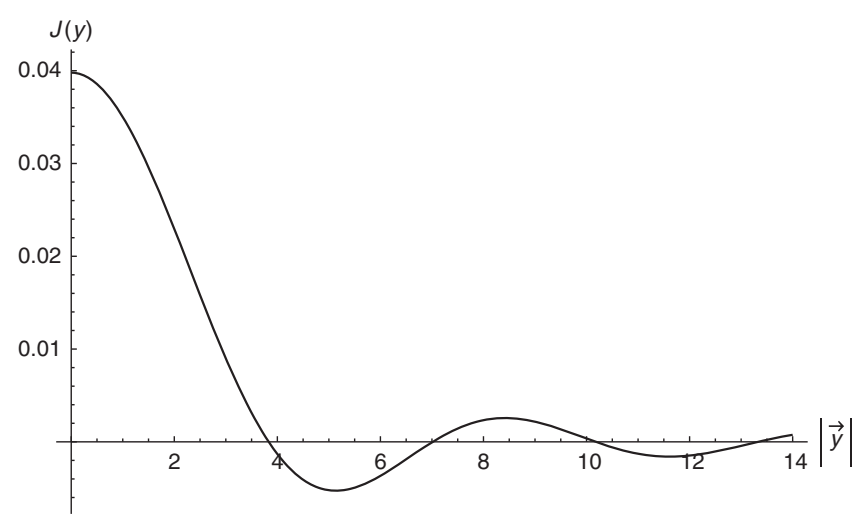

FIG. 1. Smeared version $J(y)$ of a classical source sharply localized in space $J_{c l}(y)=\delta(\mathbf{y})$. Here, we have set the deformation parameter $\kappa=1$.

can indeed be seen as a smeared version of the classical source (79). For instance, considering a classical source sharply localized in space $J_{c l}(y)=\delta(\mathbf{y})$, for which $\widetilde{J}(p)=$ $2 \pi \delta\left(p_{0}\right)$, the source $J(y)$ takes the form

$J(y)=2 \int_{0}^{\kappa} \frac{d p p}{(2 \pi)^{2} \sqrt{1-p^{2} / \kappa^{2}}} \frac{\sin (p|\mathbf{y}|)}{|\mathbf{y}|}=\frac{\kappa^{2}}{4 \pi} \frac{J_{1}(\kappa|\mathbf{y}|)}{|\mathbf{y}|}$,

where $J_{1}$ is the Bessel function of the first kind (Fig. 1).

Summarizing we have the following two pictures for the propagation of the noncommutative $\kappa$-scalar field in terms of commuting fields:

(1) Given a perturbation generated by a classical, and virtually sharply localized source $J_{c l}(y)$, the field responds by propagating through the $\kappa$-deformed Feynman propagator $i \Delta_{F}^{\kappa}(x-y)$.

(2) Given a perturbation generated by a $\kappa$-deformed source $J(y)$, the field responds by propagating through the standard Feynman propagator $i \Delta_{F}(x-y)$.

This result provides a concrete realization of the picture qualitatively outlined in [28]. There it was showed that for $\kappa$-deformed fields, the Yukawa potential between two static point sources does not diverge in the short-distance limit, and that this feature could be interpreted in terms of pointlike sources being effectively smoothed out by the UV features of the $\kappa$ deformation. This is precisely what is realized in the propagation picture (80) outlined above. Although equally interesting, we postpone an analysis of such a limitation in localizing sources in an arbitrarily small region to future studies, while, in this work, we will focus on the first picture of field propagation (78), where all the nontrivial structures due to the noncommutativity are contained in the $\kappa$-deformed Feynman propagator (77). The next step will be the explicit evaluation of the integral in (77) to analyze the spacetime behavior of $i \Delta_{F}^{\kappa}(x)$.

\section{Spacetime profile of the $\boldsymbol{\kappa}$-deformed propagator}

In order to study the spacetime properties of the $\kappa$-deformed Feynman propagator, we must first stress that in the classical basis of the $\kappa$-Poincaré algebra the action of Lorentz transformations is undeformed (unlike, e.g., the bicrossproduct basis). We indeed have that the $\kappa$-deformed Feynman propagator is manifestly Lorentz invariant

$i \Delta_{F}^{\kappa}(x-y)=i \int \frac{d^{4} p \theta\left(\kappa^{2}-p^{2}\right)}{(2 \pi)^{4} \sqrt{1-p^{2} / \kappa^{2}}} \frac{e^{i p(x-y)}}{-p^{2}-m^{2}+i \varepsilon}$,

and thus, the analysis of its spacetime behavior can be divided, as in the standard case, according to whether the spacetime separation $x^{\mu}-y^{\mu}$ is spacelike $(x-y)^{2}>0$, timelike $(x-y)^{2}<0$, or lightlike $(x-y)^{2}=0$. Let us notice, however, that the undeformed character of Lorentz transformations in the classical basis is limited to the oneparticle sector of the theory. Indeed, when one considers multiparticle states, the nontrivial coalgebra structure in (17), (19) enters the game, and the covariance of multiparticle states and observables should be assessed taking into account such deformed structures.

Before we start our analysis, let us observe that the Feynman propagator (83) can be conveniently expressed as

$$
\begin{aligned}
i \Delta_{F}^{\kappa}(x-y)= & i \int \frac{d^{3} p}{(2 \pi)^{4}} e^{i \mathbf{p}(\mathbf{x}-\mathbf{y})} \int \frac{d p_{0} \kappa \theta\left(\kappa^{2}-\mathbf{p}^{2}+p_{0}^{2}\right)}{\sqrt{\kappa^{2}-\mathbf{p}^{2}+p_{0}^{2}}} \\
& \times \frac{e^{-i p_{0}\left(x_{0}-y_{0}\right)}}{p_{0}^{2}-\omega_{\mathbf{p}}^{2}+i \varepsilon}
\end{aligned}
$$

where $\omega_{\mathbf{p}}=\sqrt{\mathbf{p}^{2}+m^{2}}$. In order to carry out the $p_{0}$ integral of this last expression, we will make use, as in the standard case, of the Cauchy's residue theorem. However, we will have to deal, besides the nontrivial $p_{0}$ range of integration, with a different singularity structure in the complex $p_{0}$ plane, as compared to the standard case.

As a first step, in view of the condition $\kappa^{2}-\mathbf{p}^{2}+p_{0}^{2}>0$, we split the range of integration in momentum space into two regions depending on whether $|\mathbf{p}|<\kappa$ or $|\mathbf{p}|>\kappa$

$$
\begin{aligned}
& A=\left\{|\mathbf{p}|<\kappa \mid p_{0} \in \mathbb{R}\right\}, \\
& B=\left\{|\mathbf{p}|>\kappa|| p_{0} \mid>\sqrt{\mathbf{p}^{2}-\kappa^{2}}\right\} .
\end{aligned}
$$

It is then possible to rewrite the expression (84) as

$$
\begin{aligned}
i \Delta_{F}^{\kappa}(x-y)= & i \int_{|\mathbf{p}|<\kappa} \frac{d^{3} p}{(2 \pi)^{4}} e^{i \mathbf{p}(\mathbf{x}-\mathbf{y})} \mathcal{I}_{\mathbf{p}}^{A}\left(x_{0}-y_{0}\right) \\
& +i \int_{|\mathbf{p}|>\kappa} \frac{d^{3} p}{(2 \pi)^{4}} e^{i \mathbf{p}(\mathbf{x}-\mathbf{y})} \mathcal{I}_{\mathbf{p}}^{B}\left(x_{0}-y_{0}\right),
\end{aligned}
$$

where we have defined the integrals 


$$
\begin{aligned}
\mathcal{I}_{\mathbf{p}}^{A}\left(x_{0}-y_{0}\right) & =\int_{-\infty}^{+\infty} \frac{d p_{0} \kappa}{\sqrt{p_{0}^{2}+\Omega_{A}^{2}}} \frac{e^{-i p_{0}\left(x_{0}-y_{0}\right)}}{p_{0}^{2}-\omega_{\mathbf{p}}^{2}+i \varepsilon}, \\
\Omega_{A} & =\sqrt{\kappa^{2}-\mathbf{p}^{2}}, \\
\mathcal{I}_{\mathbf{p}}^{B}\left(x_{0}-y_{0}\right) & =\int_{\Omega_{B}}^{\infty} \frac{d p_{0} \kappa}{\sqrt{p_{0}^{2}-\Omega_{B}^{2}}} \frac{e^{-i p_{0}\left(x_{0}-y_{0}\right)}+e^{i p_{0}\left(x_{0}-y_{0}\right)}}{p_{0}^{2}-\omega_{\mathbf{p}}^{2}+i \varepsilon}, \\
\Omega_{B} & =\sqrt{\mathbf{p}^{2}-\kappa^{2}} .
\end{aligned}
$$

As we will see, the singularity structure of $i \Delta_{F}^{\kappa}$ in the complex $p_{0}$ plane will differ in the sub-Planckian $(|\mathbf{p}|<\kappa)$ and trans-Plankian $(|\mathbf{p}|>\kappa)$ regions. Accordingly, we will divide our analysis in two parts.

\section{Region A: Sub-Planckian modes}

In the region $A$, we deal with the integral

$$
\begin{aligned}
& \mathcal{I}_{\mathbf{p}}^{A}\left(x_{0}-y_{0}\right) \\
& \quad=\int_{-\infty}^{+\infty} \frac{d p_{0} \kappa}{\sqrt{p_{0}^{2}+\Omega_{A}^{2}}} \frac{e^{-i p_{0}\left(x_{0}-y_{0}\right)}}{\left(p_{0}-\omega_{\mathbf{p}}+i \varepsilon\right)\left(p_{0}+\omega_{\mathbf{p}}-i \varepsilon\right)},
\end{aligned}
$$

where $\Omega_{A}=\sqrt{\kappa^{2}-\mathbf{p}^{2}} \in \mathbb{R}^{+}$due to the fact that here $|\mathbf{p}|<\kappa$. The square root at denominator can be written as $\sqrt{\left(p_{0}-i \Omega_{A}\right)\left(p_{0}+i \Omega_{A}\right)}$, so that the points $\pm i \Omega_{A}$ are two branch points of the square root type. As in the standard case, one can solve the integral $\mathcal{I}_{\mathbf{p}}^{A}\left(x_{0}-y_{0}\right)$ employing Cauchy's residue theorem. However, it is first necessary to cut the complex $p_{0}$ plane from $+i \Omega_{A}$ to $-i \Omega_{A}$ passing by infinity. Therefore, aside from the two undeformed simple poles in $p_{0}= \pm \omega_{\mathbf{p}} \mp i \varepsilon$, the singularity structure of the integrand in the complex $p_{0}$ plane counts two branch cuts from $+i \Omega_{A}$ to $+i \infty$ and from $-i \Omega_{A}$ to $-i \infty$.

The path in the complex plane that we use to evaluate $\mathcal{I}_{\mathbf{p}}^{A}\left(x_{0}-y_{0}\right)$ when $x_{0}>y_{0}$ is the one shown in Fig. 2; while when $x_{0}<y_{0}$, the path must be closed in the upper half plane. The result valid in both cases is

$$
\begin{aligned}
\mathcal{I}_{\mathbf{p}}^{A}\left(x_{0}-y_{0}\right)= & \frac{-2 \pi i}{\sqrt{1+m^{2} / \kappa^{2}}} \frac{e^{-i \omega_{\mathbf{p}}\left|x_{0}-y_{0}\right|}}{2 \omega_{\mathbf{p}}} \\
& -2 \int_{\Omega_{A}}^{\infty} \frac{d z \kappa}{\sqrt{z^{2}-\Omega_{A}^{2}}} \frac{e^{-z\left|x_{0}-y_{0}\right|}}{z^{2}+\omega_{\mathbf{p}}^{2}},
\end{aligned}
$$

where the standard residue at the pole is only modified by the constant multiplicative factor $\left(1+m^{2} / \kappa^{2}\right)^{-1 / 2}$, while the new term comes from the discontinuity along the branch cut on the imaginary axis.

Let us pause for a moment on what consequences the presence of these imaginary axis branch cuts could have on the scalar field propagation. Let us consider, for simplicity, the case of a $\kappa$-deformed retarded propagation of the field (for which both the simple poles are in the lower half plane)

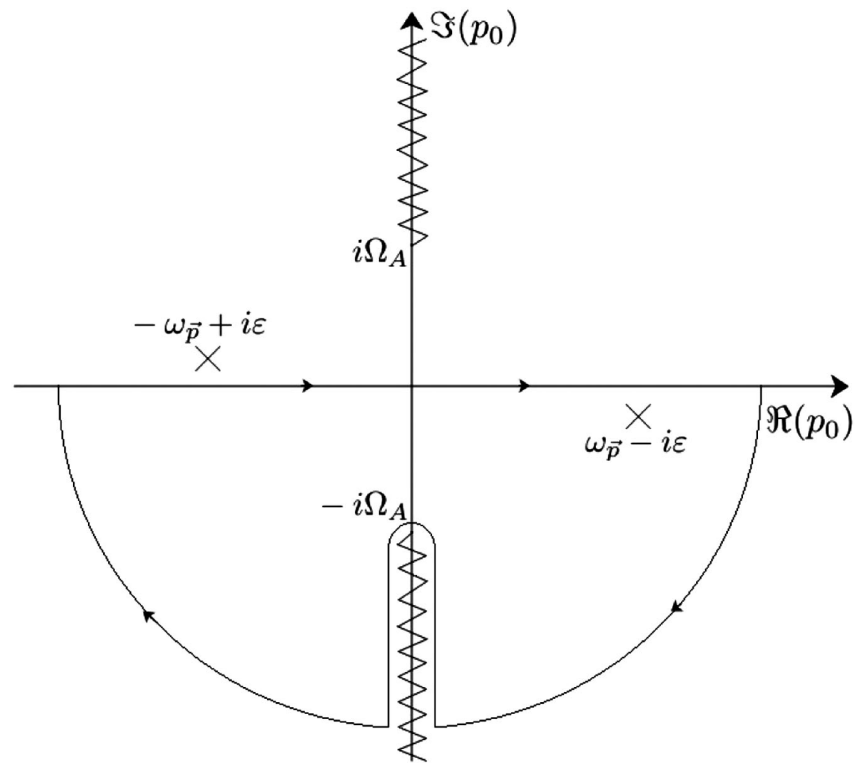

FIG. 2. Sector $A$, path in the complex $p_{0}$ plane used to evaluate $\mathcal{I}_{\mathbf{p}}^{A}\left(x_{0}-y_{0}\right)$ when $x_{0}>y_{0}$.

generated by a pointlike source $J_{c l}(y)=\delta\left(y_{0}-t\right) \delta(\mathbf{y}-\mathbf{r})$ which appears at $y_{0}=t$ and suddenly disappears. The presence of the cuts on the imaginary axis makes it impossible to define a retarded propagator $G_{R}^{\kappa}(x-y)$ that vanishes for $x_{0}<y_{0}$. As a result, the field will respond to the perturbation generated by $J_{c l}(y)$ before the source itself switches on at $y_{0}=t$. Although quite puzzling, this feature of the $\kappa$-deformed retarded propagator can be interpreted as an effect of a spacetime fuzziness determined by the noncommutativity. Indeed, in the equivalent picture related to the field propagation (80), we would have a propagation mediated by the standard retarded propagator $G_{R}(x-y)$, and a source $J(y)$ that, being a smeared version of the pointlike source $J_{c l}(y)$, results active before the time $t$. Thus, in the first picture, one has a $\kappa$-deformed retarded propagator whose advanced effects are generated by the tachyon branch cuts, while, in the second picture, one has a source smoothed out by the $\kappa$ deformation that, having support for times earlier than $t$, allows a retarded field propagation before the time $t$.

\section{Region B: Trans-Planckian modes}

The contribution to $i \Delta_{F}^{\kappa}(x-y)$ from the region $B$ is given by the integral

$$
\begin{aligned}
\mathcal{I}_{\mathbf{p}}^{B}\left(x_{0}-y_{0}\right) & =\int_{\Omega_{B}}^{+\infty} \frac{d p_{0} \kappa}{\sqrt{p_{0}^{2}-\Omega_{B}^{2}}} \frac{e^{-i p_{0}\left(x_{0}-y_{0}\right)}+e^{i p_{0}\left(x_{0}-y_{0}\right)}}{\left(p_{0}-\omega_{\mathbf{p}}+i \varepsilon\right)\left(p_{0}+\omega_{\mathbf{p}}-i \varepsilon\right)} \\
& =\mathcal{I}_{\mathbf{p}}^{B(-)}\left(x_{0}-y_{0}\right)+\mathcal{I}_{\mathbf{p}}^{B(+)}\left(x_{0}-y_{0}\right),
\end{aligned}
$$

where we have denoted with $\mathcal{I}_{\mathbf{p}}^{B( \pm)}$ the positive and negative frequency part of $\mathcal{I}_{\mathbf{p}}^{B}$. In this case, $\Omega_{B}=\sqrt{\mathbf{p}^{2}-\kappa^{2}} \in \mathbb{R}^{+}$ 

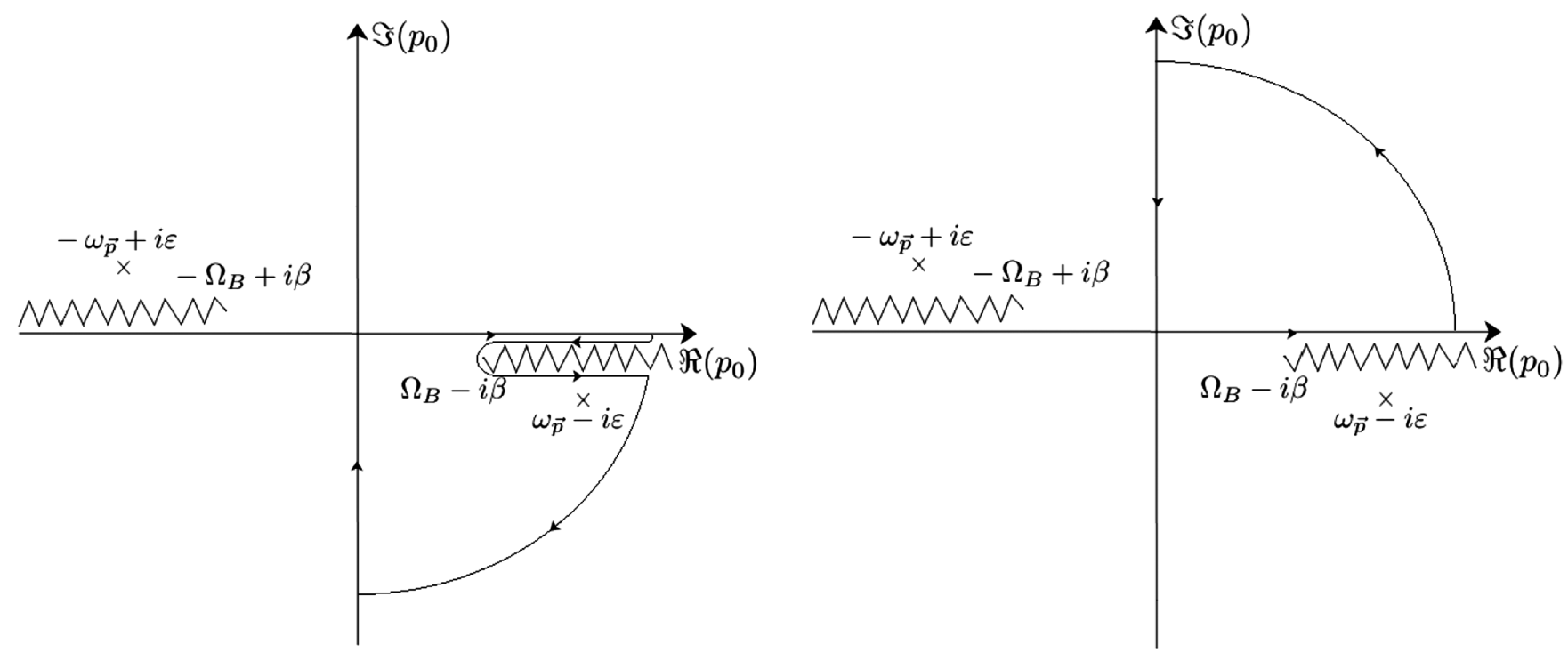

FIG. 3. Sector $B$, paths in the complex $p_{0}$ plane used to evaluate $\mathcal{I}_{\mathbf{p}}^{B(-)}\left(x_{0}-y_{0}\right)$ when $x_{0}>y_{0}$ and $x_{0}<y_{0}$, respectively. We have displaced the branch cuts by a small imaginary term in order to visually take into account the discontinuity along the cut.

due to the fact that $|\mathbf{p}|>\kappa$. Therefore, the real points $\pm \Omega_{B}$ are branch points of the square root type and, in order to solve the integrals via Cauchy's residue theorem, we have to cut the complex $p_{0}$ plane from $\Omega_{B}$ to $-\Omega_{B}$ passing by $\infty$ (see Fig. 3). We choose to focus on the negative frequency integral

$$
\begin{aligned}
& \mathcal{I}_{\mathbf{p}}^{B(-)}\left(x_{0}-y_{0}\right) \\
& \quad=\int_{\Omega_{B}}^{+\infty} \frac{d p_{0} \kappa}{\sqrt{p_{0}^{2}-\Omega_{B}^{2}}} \frac{e^{-i p_{0}\left(x_{0}-y_{0}\right)}}{\left(p_{0}-\omega_{\mathbf{p}}+i \varepsilon\right)\left(p_{0}+\omega_{\mathbf{p}}-i \varepsilon\right)},
\end{aligned}
$$

since it is then easy to extend the result to $\mathcal{I}_{\mathbf{p}}^{B(+)}\left(x_{0}-y_{0}\right)$.

The paths in the complex $p_{0}$ plane that we use to evaluate $\mathcal{I}_{\mathbf{p}}^{B(-)}$ are shown in Fig. 3 and give as a result

$$
\begin{aligned}
\mathcal{I}_{\mathbf{p}}^{B(-)}\left(x_{0}-y_{0}\right)= & -\int_{0}^{\infty} \frac{d z \kappa}{\sqrt{\Omega_{B}^{2}+z^{2}}} \frac{e^{-z\left(x_{0}-y_{0}\right)}}{z^{2}+\omega_{\mathbf{p}}^{2}} \\
& -i \int_{0}^{\Omega_{B}} \frac{d p_{0} \kappa}{\sqrt{\Omega_{B}^{2}-p_{0}^{2}}} \frac{e^{-i p_{0}\left(x_{0}-y_{0}\right)}}{p_{0}^{2}-\omega_{\mathbf{p}}^{2}} \\
& +\frac{-2 \pi i}{\sqrt{1+\frac{m^{2}}{\kappa^{2}}}} \frac{e^{-i \omega_{\mathbf{p}}\left(x_{0}-y_{0}\right)}}{2 \omega_{\mathbf{p}}}, \\
\mathcal{I}_{\mathbf{p}}^{B(-)}\left(x_{0}-y_{0}\right)= & -\int_{0}^{\infty} \frac{d z \kappa}{\sqrt{\Omega_{B}^{2}+z^{2}}} \frac{e^{z\left(x_{0}-y_{0}\right)}}{z^{2}+\omega_{\mathbf{p}}^{2}} \\
& +i \int_{0}^{\Omega_{B}} \frac{d p_{0} \kappa}{\sqrt{\Omega_{B}^{2}-p_{0}^{2}}} \frac{e^{-i p_{0}\left(x_{0}-y_{0}\right)}}{p_{0}^{2}-\omega_{\mathbf{p}}^{2}},
\end{aligned}
$$

for $x_{0}>y_{0}$ and $x_{0}<y_{0}$, respectively. From the formulas (92), the integral $\mathcal{I}_{\mathbf{p}}^{B(+)}$ can be straightforwardly evaluated.
Then, considering the Eq. (90), one obtains for the $p_{0}$ integral in the region $B$, the expression

$$
\begin{aligned}
\mathcal{I}_{\mathbf{p}}^{B}\left(x_{0}-y_{0}\right)= & \frac{-2 \pi i}{\sqrt{1+m^{2} / \kappa^{2}}} \frac{e^{-i \omega_{\mathbf{p}}\left|x_{0}-y_{0}\right|}}{2 \omega_{\mathbf{p}}} \\
& -2 \int_{0}^{\Omega_{B}} \frac{d p_{0} \kappa}{\sqrt{\Omega_{B}^{2}-p_{0}^{2}}} \frac{\sin \left(p_{0}\left|x_{0}-y_{0}\right|\right)}{p_{0}^{2}-\omega_{\mathbf{p}}^{2}} \\
& -2 \int_{0}^{\infty} \frac{d z \kappa}{\sqrt{\Omega_{B}^{2}+z^{2}}} \frac{e^{-z\left|x_{0}-y_{0}\right|}}{z^{2}+\omega_{\mathbf{p}}^{2}}
\end{aligned}
$$

Collecting now the results (89) and (93) obtained above, and plugging them in (86), we can write down the final expression of the $\kappa$-deformed Feynman propagator (84) as

$$
i \Delta_{F}^{\kappa}(x-y)=\frac{i \Delta_{F}(x-y)}{\sqrt{1+m^{2} / \kappa^{2}}}+i \Pi_{F}^{\kappa}(x-y),
$$

where $i \Delta_{F}(x-y)$ is the standard free scalar Feynman propagator

$$
i \Delta_{F}(x-y)=\int \frac{d^{3} p}{(2 \pi)^{3} 2 \omega_{\mathbf{p}}} e^{i \mathbf{p} \cdot(\mathbf{x}-\mathbf{y})} e^{-i \omega_{\mathbf{p}}\left|x_{0}-y_{0}\right|},
$$

and $i \Pi_{F}^{\kappa}(x-y)$ is given by

$$
\begin{aligned}
i \Pi_{F}^{\kappa}(x-y)= & \int_{|\mathbf{p}|<\kappa} \frac{d^{3} p}{(2 \pi)^{4}} e^{i \mathbf{p} \cdot(\mathbf{x}-\mathbf{y})} I^{A} \\
& +\int_{|\mathbf{p}|>\kappa} \frac{d^{3} p}{(2 \pi)^{4}} e^{i \mathbf{p} \cdot(\mathbf{x}-\mathbf{y})}\left(I_{1}^{B}+I_{2}^{B}\right),
\end{aligned}
$$

where, in order to express $i \Pi_{F}^{\kappa}(x-y)$ in a compact form, we have defined the integrals 


$$
\begin{aligned}
& I^{A}=-2 i \int_{\Omega_{A}}^{\infty} \frac{d z \kappa}{\sqrt{z^{2}-\Omega_{A}^{2}}} \frac{e^{-z\left|x_{0}-y_{0}\right|}}{z^{2}+\omega_{\mathbf{p}}^{2}}, \\
& I_{1}^{B}=-2 i \int_{0}^{\infty} \frac{d z \kappa}{\sqrt{\Omega_{B}^{2}+z^{2}}} \frac{e^{-z\left|x_{0}-y_{0}\right|}}{z^{2}+\omega_{\mathbf{p}}^{2}}, \\
& I_{2}^{B}=-2 i \int_{0}^{\Omega_{B}} \frac{d p_{0} \kappa}{\sqrt{\Omega_{B}^{2}-p_{0}^{2}}} \frac{\sin \left(p_{0}\left|x_{0}-y_{0}\right|\right)}{p_{0}^{2}-\omega_{\mathbf{p}}^{2}} .
\end{aligned}
$$

Notice that in the limit $\kappa \rightarrow \infty$ the $\kappa$-deformed Feynman propagator reduces to the ordinary one, as it should be. Indeed, besides the trivial constant factor $\left(1+m^{2} / \kappa^{2}\right)^{-1 / 2}$ getting to 1 , the term $i \Pi_{F}^{\kappa}(x-y)$ disappears, since the integral $I^{A}$ goes to zero, as $\Omega_{A} \rightarrow \infty$, and the trans-Planckian sector $B$ disappears. Notice also that the deformation term $i \Pi_{F}^{\kappa}(x-y)$ is a purely imaginary quantity, and thus, the real part of $i \Delta_{F}^{\kappa}(x-y)$ differs from the standard one only by the constant multiplicative factor ${ }^{10}\left(1+m^{2} / \kappa^{2}\right)^{-1 / 2}$.

Having derived the explicit expression for the $\kappa$ deformed propagator (94), we now proceed with the analysis of its spacetime behavior. As customary we will divide the discussion in three cases depending on the sign of the norm of the spacetime separation $x^{\mu}$.

\section{Lightlike separation}

When the spacetime separation $x^{\mu}$ is a null vector, i.e., we are on the light cone $x^{0}=|\mathbf{x}|$, with a Lorentz transformation, one can set $x^{\mu}=(0, \mathbf{0})$. Therefore, we consider the following $\kappa$-deformed Feynman propagator:

$$
i \Delta_{F}^{\kappa}(0)=\frac{i \Delta_{F}(0)}{\sqrt{1+m^{2} / \kappa^{2}}}+i \Pi_{F}^{\kappa}(0)
$$

The three integrals (97) that define $i \Pi_{F}^{\kappa}(0)$ can be explicitly computed as

$$
\begin{aligned}
& I^{A}=-2 i \frac{\sinh ^{-1}\left(\frac{\omega_{\mathbf{p}}}{\sqrt{\kappa^{2}-\mathbf{p}^{2}}}\right)}{\sqrt{1+m^{2} / \kappa^{2}} \omega_{\mathbf{p}}}, \\
& I_{1}^{B}=-2 i \frac{\cosh ^{-1}\left(\frac{\omega_{\mathbf{p}}}{\sqrt{\mathbf{p}^{2}-\kappa^{2}}}\right)}{\sqrt{1+m^{2} / \kappa^{2}} \omega_{\mathbf{p}}}, \quad I_{2}^{B}=0,
\end{aligned}
$$

so that, introducing spherical polar coordinates and performing the angular integration, one gets

\footnotetext{
${ }^{10}$ Which is, however, negligible if we identify $\kappa$ with the Planck energy $E_{p} \sim 10^{28} \mathrm{eV}$ and consider any of the particle masses of the standard model.
}

$$
\begin{aligned}
i \Pi_{F}^{\kappa}(0)= & \frac{-4 i}{\sqrt{1+m^{2} / \kappa^{2}}}\left[\int_{0}^{\kappa} \frac{d p}{(2 \pi)^{3}} \frac{p^{2}}{\omega_{p}} \sinh ^{-1}\left(\frac{\omega_{p}}{\sqrt{\kappa^{2}-p^{2}}}\right)\right. \\
& \left.+\int_{\kappa}^{\infty} \frac{d p}{(2 \pi)^{3}} \frac{p^{2}}{\omega_{p}} \cosh ^{-1}\left(\frac{\omega_{p}}{\sqrt{p^{2}-\kappa^{2}}}\right)\right], \quad(100)
\end{aligned}
$$

where $|\mathbf{p}|=p$.

According to (98), on the light cone, the $\kappa$-deformed Feynman propagator has the standard real quadratic divergence coming from $i \Delta_{F}(0)$ and, in addition, due to the novel contribution of $i \Pi_{F}^{\kappa}(0)$, it now exhibits also an imaginary divergent part. This imaginary contribution is due to the second integral in the square brackets of (100). Indeed, given that

$\cosh ^{-1}\left(\frac{\omega_{p}}{\sqrt{p^{2}-\kappa^{2}}}\right) \sim \frac{\sqrt{\kappa^{2}+m^{2}}}{p}+O\left(\frac{1}{p^{2}}\right), \quad p \rightarrow \infty$,

the integrand $\left[\frac{p^{2}}{\omega_{p}} \cosh ^{-1}\left(\frac{\omega_{p}}{\sqrt{p^{2}-\kappa^{2}}}\right)\right]$, for large values of $p$, approaches a constant so that the imaginary contribution of $i \Pi_{F}^{\kappa}(0)$ is linearly divergent.

\section{Spacelike separation}

For spacelike separation $x^{2}>0$, one can set $x^{0}=0$ and $|\mathbf{x}|=\sqrt{x^{2}}=r$. Taking into account the Eq. (94), the $\kappa$ deformed Feynman propagator reads

$$
i \Delta_{F}^{\kappa}(r)=\frac{i \Delta_{F}(r)}{\sqrt{1+m^{2} / \kappa^{2}}}+i \Pi_{F}^{\kappa}(r) .
$$

Given that the term $i \Pi_{F}^{\kappa}(r)$ is a pure imaginary quantity, the only contribution to real part of the $\kappa$-deformed Feynman propagator comes from $i \Delta_{F}(r)$. Such a term is real and can be expressed [59] in terms of the Hankel function of the second kind as

$\Re\left[i \Delta_{F}^{\kappa}(r)\right]=\frac{i \Delta_{F}(r)}{\sqrt{1+m^{2} / \kappa^{2}}}=\frac{-m}{8 \pi \sqrt{1+m^{2} / \kappa^{2}}} \frac{1}{r} H_{1}^{(2)}(-i m r)$.

Introducing spherical coordinates and performing the angular integration in momentum space, the imaginary part of the $\kappa$-deformed Feynman propagator (102) reads

$$
\begin{aligned}
\Im\left[i \Delta_{F}^{\kappa}(r)\right]= & i \Pi_{F}^{\kappa}(r)=\frac{-4 i}{\sqrt{1+m^{2} / \kappa^{2}}} \\
& \times\left[\int_{0}^{\kappa} \frac{d p}{(2 \pi)^{3}} \frac{p}{\omega_{p}} \sinh ^{-1}\left(\frac{\omega_{p}}{\sqrt{\kappa^{2}-p^{2}}}\right) \frac{\sin (p r)}{r}\right. \\
& \left.+\int_{\kappa}^{\infty} \frac{d p}{(2 \pi)^{3}} \frac{p}{\omega_{p}} \cosh ^{-1}\left(\frac{\omega_{p}}{\sqrt{p^{2}-\kappa^{2}}}\right) \frac{\sin (p r)}{r}\right],
\end{aligned}
$$



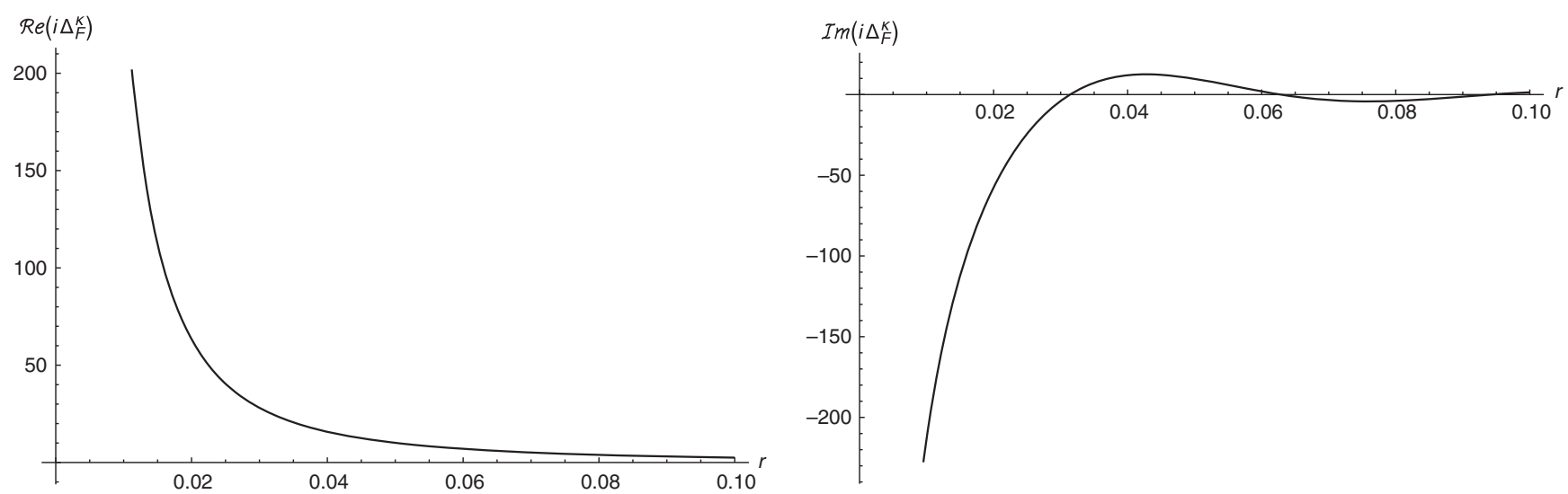

FIG. 4. Plots of the real (left) and imaginary (right) part of the $\kappa$-deformed Feynman propagator (102) for spacelike separation $x^{2}=r^{2}>0$. In the undeformed case, there is no imaginary part. The spacelike separation $r$ varies on the horizontal axis. In this simulation, we have set $\kappa=10^{2}$ and $m=1$, so that the ratio is $m / \kappa=10^{-2}$. We are looking at distances of 10 Planck lengths $\kappa^{-1}$ from the light cone, or, equivalently, at distances of $10^{-1}$ Compton wavelengths $m^{-1}$ from the light cone. In the plot of the real part, the difference between the standard and the deformed graph is not visually appreciable, being of the order of $(\mathrm{m} / \kappa)^{2}$, and the two graphs overlap.

where $|\mathbf{p}|=p$. In contrast with the standard case, the $\kappa$ deformed Feynman propagator now possesses both a real and an imaginary part given by the standard Feynman propagator $i \Delta_{F}(r)$ and by $i \Pi_{F}^{\kappa}(r)$, respectively. We proceeded to a numerical evaluation of $i \Pi_{F}^{\kappa}(r)$, and the real and the imaginary part of $i \Delta_{F}^{\kappa}(r)$ are shown in Fig. 4.

As in the undeformed case the real part of $i \Delta_{F}^{\kappa}(r)$ rapidly falls to zero with a scale set by the Compton wavelength $m^{-1}$. Indeed, the real part of $i \Delta_{F}^{\kappa}(r)$ is only modified, with respect to the standard case, by a constant multiplicative factor $\left(1+m^{2} / \kappa^{2}\right)^{-1 / 2}$. The leading correction coming from this term is of $\operatorname{order}^{11}(m / \kappa)^{2}$ which does not lead to visually appreciable changes in the first plot in Fig. 4. The imaginary part of $i \Delta_{F}^{\kappa}(r)$, absent in the undeformed case, is divergent on the light cone (in accordance with the analysis made in the previous paragraph) and falls to zero oscillating after few Planck lengths $\kappa^{-1}$ from the light cone.

The fact that the Feynman propagator is nonzero for spacelike distances is a well-known quantum mechanical tunneling phenomenon caused by the difficulty to sharply localize a particle in spacetime. However, now, in addition to the localization limit given by the particle's Compton wavelength, we also have an effect generated by the fuzzy nature of spacetime, which results in the nonvanishing additional imaginary contribution of Fig. 4 (right).

\section{Timelike separation}

When the event $x$ is at timelike separation $\left(x^{2}<0\right)$ from the origin, we can set $x^{\mu}=\left(x^{0}, \boldsymbol{0}\right)$, so that

\footnotetext{
${ }^{11}$ Here we have expanded the square root $(1+\epsilon)^{-1 / 2}$ with respect to the small parameter $\epsilon=m^{2} / \kappa^{2} \ll 1$, i.e., $(1+\epsilon)^{-1 / 2} \sim$ $1-\frac{1}{2} \epsilon+O\left((\epsilon)^{2}\right)$.
}

$$
i \Delta_{F}^{\kappa}\left(x_{0}\right)=\frac{i \Delta_{F}\left(x_{0}\right)}{\sqrt{1+m^{2} / \kappa^{2}}}+i \Pi_{F}^{\kappa}\left(x_{0}\right)
$$

The real and the imaginary contributions to $i \Delta_{F}^{\kappa}\left(x_{0}\right)$ can be identified as follows:

$$
\begin{aligned}
& \Re\left[i \Delta_{F}^{\kappa}\left(x_{0}\right)\right]=\Re\left[\frac{i m}{8 \pi \sqrt{1+m^{2} / \kappa^{2}}} \frac{1}{x_{0}} H_{1}^{(2)}\left(m x_{0}\right)\right], \\
& \Im\left[i \Delta_{F}^{\kappa}\left(x_{0}\right)\right]=\Im\left[\frac{i m}{8 \pi \sqrt{1+m^{2} / \kappa^{2}}} \frac{1}{x_{0}} H_{1}^{(2)}\left(m x_{0}\right)\right] \\
& +i \Pi_{F}^{\kappa}\left(x_{0}\right) \text {, }
\end{aligned}
$$

where we have used the fact that the undeformed Feynman propagator can be expressed in terms of Hankel functions of the second kind and that $i \Pi_{F}^{\kappa}\left(x_{0}\right)$ is a pure imaginary term.

Introducing spherical polar coordinates and performing the angular integration in momentum space, the term $i \Pi_{F}^{\kappa}\left(x_{0}\right)$ takes the form

$i \Pi_{F}^{\kappa}\left(x_{0}\right)=2\left[\int_{0}^{\kappa} \frac{d p}{(2 \pi)^{3}} p^{2} I^{A}+\int_{\kappa}^{\infty} \frac{d p}{(2 \pi)^{3}} p^{2}\left(I_{1}^{B}+I_{2}^{B}\right)\right]$

where $|\mathbf{p}|=p$ and the integrals $I^{A}, I_{1}^{B}$, and $I_{2}^{B}$ are defined in (97). This expression cannot be analytically computed; therefore, we proceeded to a numerical evaluation. The resulting real and the imaginary part of $i \Delta_{F}^{\kappa}\left(x_{0}\right)$ are shown in Fig. 5.

In Fig. 5 (right) are shown, in dashed line, the undeformed imaginary part of the propagator and, in solid line, the $\kappa$-deformed one. The effect of the $\kappa$ deformation is dominant near the light cone, where the imaginary part of $i \Delta_{F}^{\kappa}\left(x_{0}\right)$ diverges in accordance with the analysis made in 

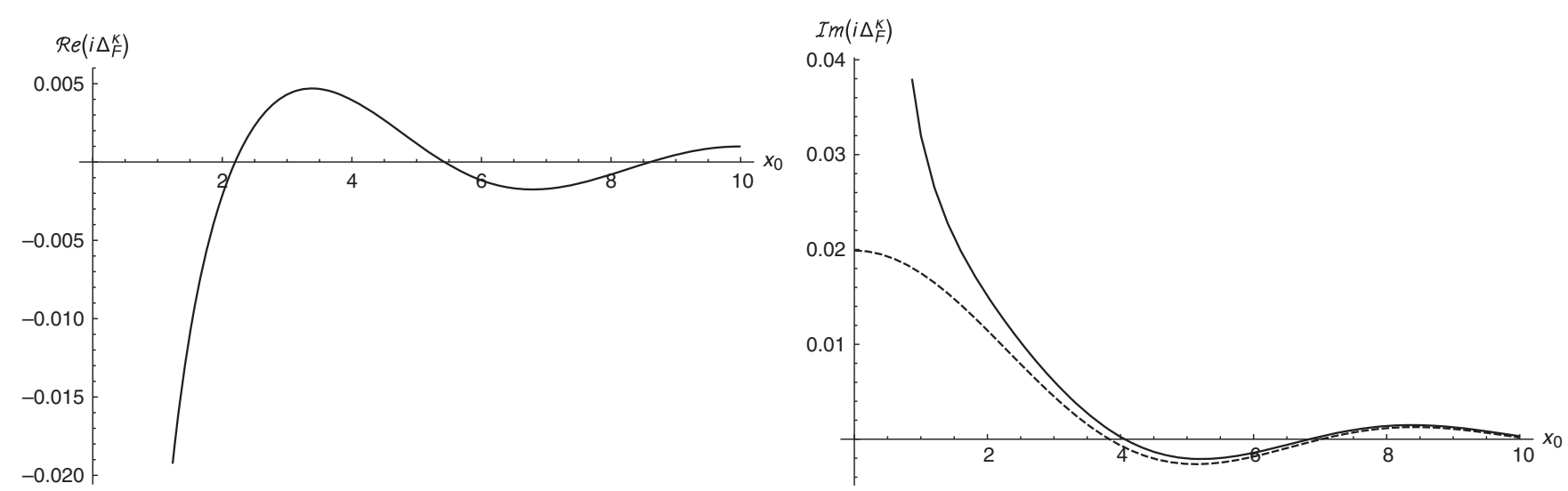

FIG. 5. Plots of the real (left) and imaginary (right) part of the $\kappa$-deformed Feynman propagator (105) for timelike separation. The timelike interval $x_{0}$ varies on the horizontal axis. In this simulation, we have set $\kappa=10^{2}$ and $m=1$, so that the ratio is $m / \kappa=10^{-2}$. Here we are looking at distances of $10^{3}$ Planck lengths $\kappa^{-1}$ from the light cone, or, equivalently, at distances of 10 Compton wavelengths $m^{-1}$ from the light cone. In the figure on the right are plotted both the imaginary part of $i \Delta_{F}^{\kappa}\left(x_{0}\right)$ (solid line) and the ordinary undeformed imaginary part of $i \Delta_{F}\left(x_{0}\right)$ (dashed line), while, in the plot of the real part on the left, the difference between the standard and the deformed graph is not visually appreciable, being of the order of $(\mathrm{m} / \kappa)^{2}$, and the two graphs overlap.

the previous paragraphs. For large timelike distances, the $\kappa$ deformed Feynman propagator approaches the standard one, so that it decreases in amplitude while oscillating according to the asymptotic behavior,

$$
i \Delta_{F}\left(x_{0}\right) \sim \operatorname{const}\left(x_{0}^{2}\right)^{-\frac{3}{4}} e^{-i m\left|x_{0}\right|}, \quad\left|x_{0}\right| \rightarrow \infty .
$$

The scale of the oscillations, inside both the future and past light cone, is set by the Compton wavelengths $m^{-1}$.

Summarizing, $\kappa$ deformations modify the real part of the Feynman propagator only by a constant multiplicative factor $\left(1+m^{2} / \kappa^{2}\right)^{-1 / 2}$. Conversely, the imaginary part of the $\kappa$-deformed Feynman propagator is $\left(1+m^{2} / \kappa^{2}\right)^{-1 / 2}$ times the undeformed one plus an additional contribution $i \Pi_{F}^{\kappa}$. This contribution is divergent on the light cone, as can be seen from the Figs. 4 and 5, for spacelike distances falls to zero oscillating after few Planck length from the light cone, and for timelike distances modifies the undeformed propagator as in Fig. 5 (right).

Before concluding this section, we would like to note that, in spite of the prominent role that the Feynman propagator plays in the standard local quantum field theory, very few attempts addressing the generalization of the Feynman propagator to noncommutative field theory on $\kappa$ Minkowski have appeared in literature. Most of the earlier attempts to the study of the $\kappa$-deformed propagator remained just at an exploratory level due to the poorly understood structure of $\kappa$-deformed momentum space at the time. This is the case, for example, of [60] and [61] where the authors introduced a $\kappa$-deformed Feynman propagator and a Pauli-Jordan function by analogy with the undeformed case, simply by replacing the standard relativistic energy-momentum dispersion relation with the $\kappa$-deformed Casimir similar to (21). More recently, a study of the PauliJordan function taking into account the nontrivial de Sitter geometry of $\kappa$-deformed momentum space has appeared in [62]. The approach taken by the authors is to define a field operator and an algebra of creation and annihilation operators, so that the commutator of the fields can be used to derive the Pauli-Jordan via its vacuum expectation value. The main drawback of the canonical approach adopted in [62] is that it relies on the definition of an algebra of creation and annihilation operator which does not take into account the known difficulties in dealing with multiparticle states in a $\kappa$-deformed context (see, e.g., $[63,64]$ ). Moreover, using the field operator defined in [62] to construct a $\kappa$-deformed Feynman propagator, one would have that only the simple poles, coming from the delta function of the Casimir, will contribute to the propagator. Our analysis instead shows that the Feynman propagator, in order to be a Green's function of the $\kappa$-Klein-Gordon equation, must contain also the contributions from the branch cuts.

\section{FROM NONCOMMUTATIVE TO NONLOCAL FIELDS}

In the previous section, we have seen how the nontrivial features of $\kappa$-deformed propagation (80) can be understood in terms of an effective spacetime fuzziness in the UV. We also pointed out how the deformed field propagation can be equivalently viewed as a propagation on classical Minkowski spacetime, i.e., with no limitations in localizing a source, mediated by the $\kappa$-deformed Feynman propagator (77).

In this section, we show how such interpretation of $\kappa$-deformed propagation, based on a classical Minkowski spacetime, can be formulated in terms of a nonlocal field theory. We will also show how the $\kappa$-deformed Feynman propagator can be related to the vacuum expectation values of products of this nonlocal field operator. We will find that 
the standard relation between the Feynman propagator and the time-ordered two-point function holds only in the subPlanckian sector, while, for trans-Planckian momenta, the $\kappa$-deformed Feynman propagator will be shown to have the form of the Hadamard (anticommutator) two-point function. We will comment on a possible physical interpretation of such feature at the end of this section.

As observed in [31,32], the free $\kappa$-Minkowski scalar field theory can be recast in the form of a nonlocal scalar field theory on ordinary Minkowski space. In these works, it was shown how the nonlocal character of the $\kappa$-Minkowski theories, already noticed in [60] for the bicrossproduct basis, is present also for the classical basis, and it is encoded in a nonlocal star product. Let us notice that the nonlocal character of the $\kappa$-Minkowski free scalar action ${ }^{12}$ could have already been noticed by considering its expression in momentum space (51) and the explicit form of the Fourier transform (45), so that (51) can be written as

$S_{\text {free }}=\int d^{4} x d^{4} x^{\prime} \phi^{*}(x)\left(-\square+m^{2}\right) \phi\left(x^{\prime}\right) V\left(x, x^{\prime}\right)$,

where the nonlocal term $V\left(x, x^{\prime}\right)$ is given by

$V\left(x, x^{\prime}\right)=\int \frac{d^{4} p \theta\left(\kappa^{2}-p^{2}\right)}{(2 \pi)^{4}} \sqrt{1-p^{2} / \kappa^{2}} e^{-i p\left(x^{\prime}-x\right)}$.

Alternatively, making use of the classical basis Weyl map $\Omega_{c}$ and its associated star product $\star$ (see Sec. II C), the $\kappa$-Poincaré invariant action (49) can be expressed as

$$
\begin{aligned}
S_{\text {free }}= & \int d^{4} x\left[\left(\partial_{\mu} \phi\right)^{\dagger} \star\left(\partial^{\mu} \phi\right)+m^{2} \phi^{\dagger} \star \phi\right] \\
= & \int d^{4} x\left[\left(\partial_{\mu} \phi\right)^{*} \sqrt{1+\square / \kappa^{2}}\left(\partial^{\mu} \phi\right)\right. \\
& \left.+m^{2} \phi^{*} \sqrt{1+\square / \kappa^{2}} \phi\right] .
\end{aligned}
$$

This action is manifestly invariant under $\kappa$-Poincaré trasformations which in the classical basis, as mentioned above, are just the standard ones. Taking the formal series expansion in powers of the d'Alembertian for the star product term, the action has infinitely many derivatives

\footnotetext{
${ }^{12}$ It should be stressed that the type of nonlocality associated to the noncommutative $\star$ product (43) and reflected in a nonlocal field action is fundamentally different from the nonlocal effects characterizing the kinematics of models with curved momentum space within the framework of relative locality [65]. Such effects refer to the crossings of worldlines of classical relativistic point particles. Some formulations of classical $\kappa$-deformed kinematics exhibit relative locality features [66,67] (see also [68] for a model which implements relative locality effects without $\kappa$-deformations), and it is likely that at a field theoretic level these are associated to the nonlocal action of $\kappa$-deformed translation generators explored, e.g., in [69].
}

$S_{\text {free }}=\int d^{4} x \sum_{n=0}^{\infty} a_{n}\left[\partial_{\mu} \phi^{*} \square^{n} \partial^{\mu} \phi+m^{2} \phi^{*} \square^{n} \phi\right]$,

where $a_{n} \propto \kappa^{-2 n}$. Varying the action (112) with respect to the field and its complex conjugate, we get

$$
\sum_{n=0}^{\infty} a_{n}\left[-\square^{n+1}+m^{2} \square^{n}\right] \phi=0
$$

and similarly for $\phi^{*}$, from which, summing up the series, one obtains the nonlocal equation of motion

$$
\sqrt{1+\square / \kappa^{2}}\left(\square-m^{2}\right) \phi=0,
$$

and an identical one for the complex conjugate field $\phi^{*}$. These equations of motion involve nonlocal pseudodifferential operators; specifically, they contain fractional powers of the d'Alembertian. ${ }^{13}$ In order to solve them, we will make use of the methods developed in [73-75] and employed, for example, in [76] to study the nonlocal effective field theory emerging in a "mesoscopic" regime of casual sets. The general form of a Lorentz invariant nonlocal pseudodifferential equation is

$$
f(\square) \phi=0,
$$

where, as for the equation of motion (113), the function $f(\square)$ cannot be expanded in a finite series. A general solution of (115) can be written as

$\phi(x)=\frac{1}{2 \pi i} \int \frac{d^{3} p}{(2 \pi)^{3 / 2}} \int_{\sum_{i} \Gamma_{i}} d p_{0} e^{i p x}\left[\frac{1}{f\left(-p^{2}\right)}\right] a\left(p_{0}, \mathbf{p}\right)$,

where $a\left(p_{0}, \mathbf{p}\right)$ is an entire analytic function, i.e., a complex-valued function that is holomorphic at all points over the whole complex plane, and the $\Gamma_{i}$ 's are paths that encircle, in the complex $p_{0}$ plane, all the singularities of $1 / f\left(-p^{2}\right)$. Indeed, rewriting the solution (116) as

\footnotetext{
${ }^{13}$ In field theories based on nonlocal d'Alembertians, one is usually concerned with the presence of unstable modes which can spoil the theory, for example, by leading to violations of unitarity [70]. For what concerns our equation of motion (114) for $\kappa^{2}-p^{2}<0$, the nonlocal factor $\sqrt{\kappa^{2}+\square}$ becomes a purely imaginary quantity, and thus, in principle, some instabilities are expected [71,72]. Looking at this nonlocal model as effectively representing a $\kappa$-deformed theory however, we see that the de Sitter geometry of the space of four momenta naturally imposes the condition $\kappa^{2}-p^{2}>0$. This is obvious if one looks at the embedding coordinates defined in (7) and notices that $\sqrt{\kappa^{2}-p^{2}}$ corresponds to the embedding coordinate $P_{4}$ and thus is real. This shows that in the $\kappa$-deformed theory the offending fourmomentum region $\kappa^{2}-p^{2}<0$ is excluded from the outset.
} 


$$
\phi(x)=\int \frac{d^{3} p}{(2 \pi)^{3 / 2}} e^{i \mathbf{p} \cdot \mathbf{x}} \phi_{\mathbf{p}}\left(x_{0}\right),
$$

where

$\phi_{\mathbf{p}}\left(x_{0}\right)=\frac{1}{2 \pi i} \int_{\sum_{i} \Gamma_{i}} d p_{0} e^{-i p_{0} x_{0}}\left[\frac{1}{f\left(-p^{2}\right)}\right] a\left(p_{0}, \mathbf{p}\right)$,

the condition (115) becomes

$$
f\left(-\partial_{0}^{2}-\mathbf{p}^{2}\right) \phi_{\mathbf{p}}\left(x_{0}\right)=0,
$$

and when we apply the operator $f\left(-\partial_{0}^{2}-\mathbf{p}^{2}\right)$ on (118), a factor $f\left(-p^{2}\right)$ that cancels all the poles and cuts is produced inside the integral, so that the paths $\Gamma_{i}$ can now be deformed to a point giving vanishing contributions. In our case, the function $f\left(-p^{2}\right)$ can be read off the $\kappa$-deformed equations of motion (114) and is given by

$$
f_{\kappa}\left(-p^{2}\right)=\sqrt{1-p^{2} / \kappa^{2}}\left(-p^{2}-m^{2}\right) .
$$

An important point to notice is that the $\kappa$-deformed contribution $\sqrt{1-p^{2} / \kappa^{2}}$ to the function $f_{\kappa}\left(-p^{2}\right)$ is just the term $\left|p_{4}\right| / \kappa$ appearing at the denominator of the $\kappa$-Poincaré momentum space integration measure $d \bar{\mu}(p)=$ $d^{4} p \theta\left(\kappa^{2}-p^{2}\right) \kappa /\left|p_{4}\right|$. Accordingly, the isolated singularities and branch cuts associated with the function $1 / f_{\kappa}\left(-p^{2}\right)$ are just the ones we have already seen in Sec. III C when solving the $p_{0}$ integral of the $\kappa$-deformed Feynman propagator. We will thus split the analysis of the solutions associated to (114) in two parts depending on whether one considers the sub-Planckian or the trans-Planckian sector. The position of the branch cuts in the complex $p_{0}$ plane are different in these two case; a cut on the imaginary $p_{0}$ axis when $|\mathbf{p}|<\kappa$ and a cut on the real $p_{0}$ axis when $|\mathbf{p}|>\kappa$ [see Figs. 2 and 3]. The solution (117) can thus be rewritten as

$$
\begin{aligned}
\phi(x)= & \phi^{A}(x)+\phi^{B}(x)=\int_{|\mathbf{p}|<\kappa} \frac{d^{3} p}{(2 \pi)^{3 / 2}} e^{i \mathbf{p} \cdot \mathbf{x}} \phi_{\mathbf{p}}^{A}\left(x_{0}\right) \\
& +\int_{|\mathbf{p}|>\kappa} \frac{d^{3} p}{(2 \pi)^{3 / 2}} e^{i \mathbf{p} \cdot \mathbf{x}} \phi_{\mathbf{p}}^{B}\left(x_{0}\right),
\end{aligned}
$$

and similarly for $\phi^{*}(x)$. Our goal in the next Section will be to find a relationship between the $\kappa$-deformed Feynman propagator found in Sec. III and vacuum expectation values of the field operator (121) above. Let us recall that, in analogy with (121), the propagator (86) exhibits a similar splitting in subPlankian and trans-Planckian contributions

$$
\begin{aligned}
i \Delta_{F}^{\kappa}(x)= & \left.i \Delta_{F}^{\kappa}(x)\right|_{A}+\left.i \Delta_{F}^{\kappa}(x)\right|_{B}=i \int_{|\mathbf{p}|<\kappa} \frac{d^{3} p}{(2 \pi)^{4}} e^{i \mathbf{p} \cdot \mathbf{x}} \mathcal{I}_{\mathbf{p}}^{A}\left(x_{0}\right) \\
& +i \int_{|\mathbf{p}|>\kappa} \frac{d^{3} p}{(2 \pi)^{4}} e^{i \mathbf{p} \cdot \mathbf{x}} \mathcal{I}_{\mathbf{p}}^{B}\left(x_{0}\right)
\end{aligned}
$$

due to the restriction imposed by the condition $\kappa^{2}-p^{2}>0$ on momentum space. In particular, we should notice that, since in the sub-Planckian region $A$ the $p_{0}$ range of integration in the propagator remains unaffected [see (85)], we expect a standard relation between $\left.i \Delta_{F}^{\kappa}(x)\right|_{A}$ and the timeordered two-point function of $\phi^{A}$. The same, however, can not be said for the trans-Planckian sector $B$. Here, the condition $\kappa^{2}-p^{2}>0$ deforms the $p_{0}$ range of integration $\left(\left|p_{0}\right|>\Omega_{B}\right)$, so we should expect a different combination of two-point functions of $\phi^{B}$ to be related to $\left.i \Delta_{F}^{\kappa}(x)\right|_{B}$. In what follows, we will study this issue in detail.

\section{THE $\kappa$-DEFORMED PROPAGATOR AS A NONLOCAL TWO-POINT FUNCTION}

In this section, we characterize the $\kappa$-deformed Feynman propagator in terms of the vacuum expectation values of the nonlocal complex scalar field on ordinary Minkowski spacetime introduced in the previous section. In order to do so we proceed to quantize the nonlocal field (117) using the techniques first developed in [73]. Once again, we focus separately on the sub-Planckian and trans-Planckian cases.

\section{A. Sub-Planckian momenta: Time-ordered two-point function}

In the region of sub-Planckian momenta, the function $f_{\kappa}$ of the Eq. (120) takes the form

$$
f_{\kappa}\left(p_{0}, \mathbf{p}\right)=\frac{2 \pi i}{\kappa} \sqrt{p_{0}^{2}+\Omega_{A}^{2}}\left(p_{0}^{2}-\omega_{\mathbf{p}}^{2}\right),
$$

where, for future convenience, we have incorporated also the term $2 \pi i$ in the definition of $f_{\kappa}$ and, as in Sec. III, $\Omega_{A}=$ $\sqrt{\kappa^{2}-\mathbf{p}^{2}}$ and $\omega_{\mathbf{p}}=\sqrt{\mathbf{p}^{2}+m^{2}}$. Considering Eqs. (118), (121), and (123), it is then possible to express the restriction of the field $\phi$ to the region $A$, taking into account the singularity structure of $1 / f_{\kappa}$, as

$$
\phi^{A}(x)=\int_{|\mathbf{p}|<\kappa} \frac{d^{3} p}{(2 \pi)^{3 / 2}} e^{i \mathbf{p} \cdot \mathbf{x}} \phi_{\mathbf{p}}^{A}\left(x_{0}\right),
$$

with

$\phi_{\mathbf{p}}^{A}\left(x_{0}\right)=\frac{1}{2 \pi i} \sum_{i=1}^{2} \int_{\Gamma_{i}^{A}+\gamma_{i}^{A}} \frac{d p_{0} \kappa}{\sqrt{p_{0}^{2}+\Omega_{A}^{2}}} \frac{e^{-i p_{0} x_{0}}}{p_{0}^{2}-\omega_{\mathbf{p}}^{2}} a\left(p_{0}, \mathbf{p}\right)$,

where the integration contours $\Gamma_{i}^{A}$ and $\gamma_{i}^{A}$ are shown in Fig. 6. We can write (125) explicitly as 


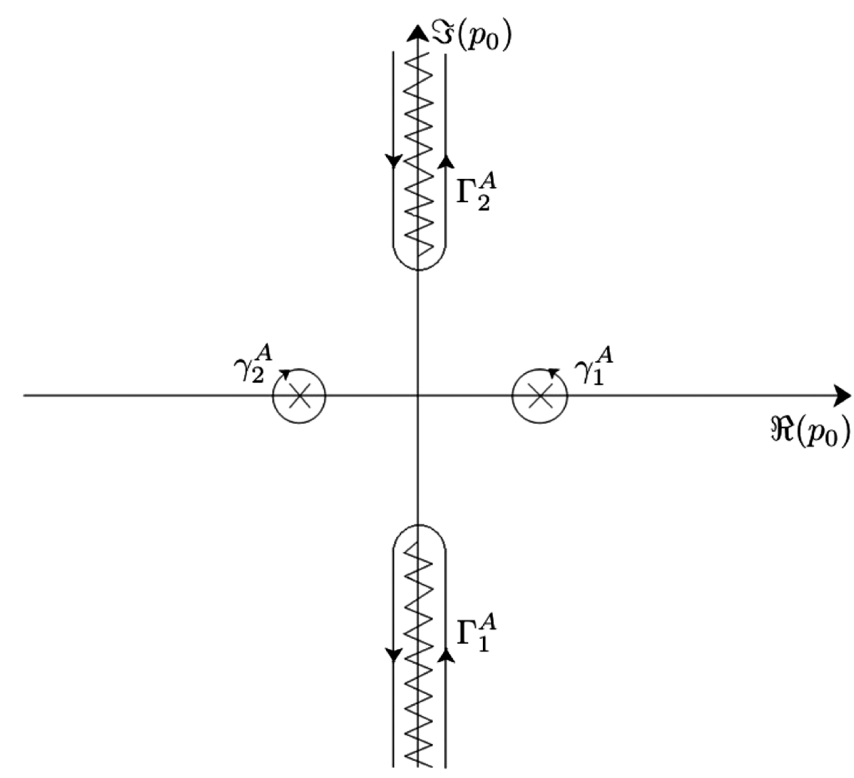

FIG. 6. Singularity structure in the region $A$. There are two simple poles at $p_{0}= \pm \omega_{\mathbf{p}}$ and two branch cuts on the imaginary axis, one from $i \Omega_{A}$ to $i \infty$ and one from $-i \Omega_{A}$ to $-i \infty$.

$$
\begin{aligned}
\phi_{\mathbf{p}}^{A}\left(x_{0}\right)= & \int_{-i \infty}^{-i \Omega_{A}} d p_{0} \Delta_{\Gamma_{A}}\left[\frac{1}{f_{\kappa}\left(p_{0}, \mathbf{p}\right)}\right] e^{-i p_{0} x_{0}} a\left(p_{0}, \mathbf{p}\right) \\
& +\int_{-i \infty}^{-i \Omega_{A}} d p_{0} \Delta_{\Gamma_{A}}\left[\frac{1}{f_{\kappa}\left(p_{0}, \mathbf{p}\right)}\right] e^{i p_{0} x_{0}} a\left(-p_{0}, \mathbf{p}\right) \\
& +\frac{1}{\sqrt{1+m^{2} / \kappa^{2}}} \frac{e^{-i \omega_{\mathbf{p}} x_{0}}}{2 \omega_{\mathbf{p}}} a\left(\omega_{\mathbf{p}}, \mathbf{p}\right) \\
& +\frac{1}{\sqrt{1+m^{2} / \kappa^{2}}} \frac{e^{i \omega_{\mathbf{p}} x_{0}}}{2 \omega_{\mathbf{p}}} a\left(-\omega_{\mathbf{p}}, \mathbf{p}\right)
\end{aligned}
$$

where the functional $\Delta_{\Gamma_{A}}\left[1 / f_{\kappa}\left(p_{0}, \mathbf{p}\right)\right]$ is the discontinuity functional at the branch cut of $1 / f_{\kappa}$, which in our case is just

$$
\Delta_{\Gamma_{A}}\left[\frac{1}{f_{\kappa}\left(p_{0}, \mathbf{p}\right)}\right]=-\frac{2}{2 \pi i} \frac{\kappa}{\sqrt{p_{0}^{2}+\Omega_{A}^{2}}} \frac{1}{p_{0}^{2}-\omega_{\mathbf{p}}^{2}} .
$$

The quantum counterpart of the field (126) is obtained by promoting the entire analytic coefficients $a(p)$ and $a(-p)$ to annihilation and creation operators, respectively. Since we are dealing with a complex field, the function $a(p)$ will be promoted to the annihilation operator for particles while $a(-p)$ to the creation operator $b^{\dagger}(p)$ of antiparticles. Following [73], we note that, in order to have a consistent quantization scheme, the creation and annihilation operators must satisfy the commutation rules

$$
\begin{aligned}
& \Delta\left[\frac{1}{f_{\kappa}\left(-p^{2}\right)}\right]\left[a(p), a^{\dagger}(q)\right]=\delta(p-q), \\
& \Delta\left[\frac{1}{f_{\kappa}\left(-p^{2}\right)}\right]\left[b(p), b^{\dagger}(q)\right]=\delta(p-q),
\end{aligned}
$$

with all the other commutators being zero. The discontinuity functional $\Delta\left[1 / f_{\kappa}\left(-p^{2}\right)\right]$ involved in the commutation relations (128) obviously depends on which singularity one considers; for instance, the discontinuity functional associated to the branch cut is the one appearing in (127), while for the simple pole one has

$$
\begin{aligned}
\Delta_{\gamma_{A}}\left[\frac{1}{f_{\kappa}\left(-p^{2}\right)}\right]= & \lim _{\varepsilon \rightarrow 0}\left[\left(\frac{1}{f_{\kappa}\left(\left(p_{0}+i \varepsilon\right)^{2}-\mathbf{p}^{2}\right)}\right.\right. \\
& \left.\left.-\frac{1}{f_{\kappa}\left(\left(p_{0}-i \varepsilon\right)^{2}-\mathbf{p}^{2}\right)}\right)\left.\right|_{\gamma_{A}}\right],
\end{aligned}
$$

and with simple manipulations

$$
\begin{aligned}
\Delta_{\gamma_{A}}\left[\frac{1}{f_{\kappa}\left(-p^{2}\right)}\right] & =\frac{1}{2 \pi i} \frac{2 \pi i}{\sqrt{1-p^{2} / \kappa^{2}}} \lim _{\varepsilon \rightarrow 0}\left(\frac{1}{\pi} \frac{\varepsilon}{\varepsilon^{2}+\left(-p^{2}-m^{2}\right)^{2}}\right) \\
& =\frac{\delta\left(p^{2}+m^{2}\right)}{\sqrt{1+m^{2} / \kappa^{2}}} .
\end{aligned}
$$

The algebra of the annihilation and creation operators for the field excitations with four momentum associated to the simple pole are then simply $\sqrt{1+m^{2} / \kappa^{2}}$ times the usual ones

$$
\begin{aligned}
& {\left[a\left(\omega_{\mathbf{p}}, \mathbf{p}\right), a^{\dagger}\left(\omega_{\mathbf{q}}, \mathbf{q}\right)\right]=2 \omega_{\mathbf{p}} \sqrt{1+m^{2} / \kappa^{2}} \delta(\mathbf{p}-\mathbf{q}),} \\
& {\left[b\left(\omega_{\mathbf{p}}, \mathbf{p}\right), b^{\dagger}\left(\omega_{\mathbf{q}}, \mathbf{q}\right)\right]=2 \omega_{\mathbf{p}} \sqrt{1+m^{2} / \kappa^{2}} \delta(\mathbf{p}-\mathbf{q}),}
\end{aligned}
$$

while for excitations with four momentum belonging to the cut, one obtains

$$
\begin{aligned}
& {\left.\left[a(p), a^{\dagger}(q)\right]\right|_{p, q \in \Gamma_{A}}=\frac{\delta(p-q)}{\Delta_{\Gamma_{A}}\left[1 / f\left(-p^{2}\right)\right]},} \\
& {\left.\left[b(p), b^{\dagger}(q)\right]\right|_{p, q \in \Gamma_{A}}=\frac{\delta(p-q)}{\Delta_{\Gamma_{A}}\left[1 / f\left(-p^{2}\right)\right]} .}
\end{aligned}
$$

We now have all the tools needed to compute the timeordered two-point functions in the region $\mathrm{A}$ as

$$
\begin{aligned}
\langle 0| & T\left\{\phi^{A}(x)\left(\phi^{A}(y)\right)^{*}\right\}|0\rangle \\
= & \theta\left(x_{0}-y_{0}\right) \int_{|\mathbf{p}|,|\mathbf{q}|<\kappa} \frac{d^{3} p d^{3} q}{(2 \pi)^{3}} e^{i \mathbf{p} \cdot \mathbf{x}} e^{-i \mathbf{q} \cdot \mathbf{y}} \\
& \times\left\langle 0\left|\phi_{\mathbf{p}}^{A}\left(x_{0}\right)\left(\phi_{\mathbf{q}}^{A}\left(y_{0}\right)\right)^{*}\right| 0\right\rangle \\
& +\theta\left(y_{0}-x_{0}\right) \int_{|\mathbf{p}|,|\mathbf{q}|<\kappa} \frac{d^{3} p d^{3} q}{(2 \pi)^{3}} e^{-i \mathbf{p} \cdot \mathbf{x}} e^{i \mathbf{q} \cdot \mathbf{y}} \\
& \times\left\langle 0\left|\left(\phi_{\mathbf{q}}^{A}\left(y_{0}\right)\right)^{*} \phi_{\mathbf{p}}^{A}\left(x_{0}\right)\right| 0\right\rangle,
\end{aligned}
$$

and, making use of the Eqs. (126), (131) and (132), we get ${ }^{14}$

\footnotetext{
${ }^{14}$ In deriving the integral inside the square brackets, we have made the changes of variable $p_{0} \rightarrow \pm i z$.
} 


$$
\begin{aligned}
\langle 0| T & \left\{\phi^{A}(x)\left(\phi^{A}(y)\right)^{*}\right\}|0\rangle \\
= & \int_{|\mathbf{p}|<\kappa} \frac{d^{3} p}{(2 \pi)^{3}} e^{i \mathbf{p} \cdot(\mathbf{x}-\mathbf{y})}\left[\frac{e^{-i \omega_{\mathbf{p}}\left|x_{0}-y_{0}\right|}}{2 \omega_{\mathbf{p}} \sqrt{1+m^{2} / \kappa^{2}}}\right. \\
& \left.+\frac{2}{2 \pi i} \int_{\Omega_{A}}^{\infty} \frac{d z \kappa}{\sqrt{z^{2}-\Omega_{A}^{2}}} \frac{e^{-z\left|x_{0}-y_{0}\right|}}{z^{2}+\omega_{\mathbf{p}}^{2}}\right] .
\end{aligned}
$$

The term in square brackets is just $i / 2 \pi$ times the integral $\mathcal{I}_{\mathbf{p}}^{A}$ defined in (89). Accordingly, this expression is equal to the restriction $\left.i \Delta_{F}^{\kappa}(x-y)\right|_{A}$ of the $\kappa$-deformed Feynman propagator to the region of sub-Planckian momenta, as can be easily seen confronting the relations (122) and (134),

$$
\begin{aligned}
\left\langle 0\left|T\left\{\phi^{A}(x)\left(\phi^{A}(y)\right)^{*}\right\}\right| 0\right\rangle & =i \int_{|\mathbf{p}|<\kappa} \frac{d^{3} p}{(2 \pi)^{4}} e^{i \mathbf{p} \cdot(\mathbf{x}-\mathbf{y})} \mathcal{I}_{\mathbf{p}}^{A}\left(x_{0}-y_{0}\right) \\
& \left.\equiv i \Delta_{F}^{\kappa}(x-y)\right|_{A} .
\end{aligned}
$$

This shows that for sub-Planckian modes, as expected, no changes occur in the standard relation between the Feynman propagator and the vacuum expectation values of the $\mathrm{T}$ product of fields.

\section{B. Trans-Planckian momenta: The Hadamard function}

We now derive the combination of nonlocal two-point functions which reproduces the restriction $\left.i \Delta_{F}^{\kappa}(x-y)\right|_{B}$ of the $\kappa$-deformed Feynman propagator to the region of transPlanckian momenta. Let us start from the $\kappa$-deformed Feynman propagator

$i \Delta_{F}^{\kappa}(x-y)=i \int \frac{d^{4} p \theta\left(\kappa^{2}-p^{2}\right)}{(2 \pi)^{4} \sqrt{1-p^{2} / \kappa^{2}}} \frac{e^{i p(x-y)}}{p_{0}^{2}-\omega_{\mathbf{p}}^{2}+i \varepsilon}$,

which we can rewrite using the integral expression for the step function $\theta\left(\kappa^{2}-p^{2}\right)$ as

$$
\begin{aligned}
i \Delta_{F}^{\kappa}(x-y)= & i \int_{-\kappa^{2}}^{+\infty} d \mu^{2} \int \frac{d^{4} p \delta\left(p^{2}+\mu^{2}\right)}{(2 \pi)^{4} \sqrt{1-p^{2} / \kappa^{2}}} \\
& \times \frac{e^{i p(x-y)}}{p_{0}^{2}-\omega_{\mathbf{p}}^{2}+i \varepsilon} .
\end{aligned}
$$

The restriction to spatial momenta bigger than $\kappa$ is given by

$$
\begin{aligned}
\left.i \Delta_{F}^{\kappa}(x-y)\right|_{B}= & i \int_{-\kappa^{2}}^{+\infty} d \mu^{2} \int_{|\mathbf{p}|>\kappa} \frac{d^{4} p \delta\left(p^{2}+\mu^{2}\right)}{(2 \pi)^{4} \sqrt{1-p^{2} / \kappa^{2}}} \\
& \times \frac{e^{i p(x-y)}}{p_{0}^{2}-\omega_{\mathbf{p}}^{2}+i \varepsilon} .
\end{aligned}
$$

For trans-Planckian momenta, the delta function $\delta\left(p_{15}^{2}+\mu^{2}\right)$ can be expanded with respect to the roots of $p_{0}$ as ${ }^{15}$

\footnotetext{
${ }^{15}$ Indeed, it is only for $|\mathbf{p}|>\kappa$ that $\omega_{\mathbf{p}}^{\mu} \in \mathbb{R}$ for all $\mu^{2} \in\left[-\kappa^{2},+\infty\right)$.
}

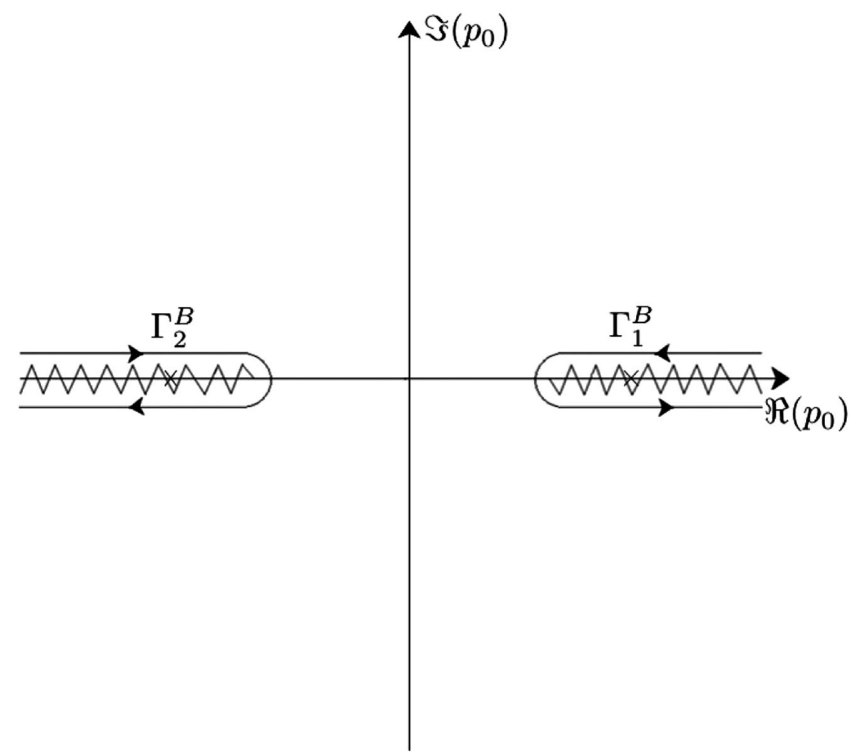

FIG. 7. Singularity structure in the region $B$. There are two branch cuts on the real axis, one from $\Omega_{B}$ to $+\infty$ and one from $-\Omega_{B}$ to $-\infty$. The two simple poles at $p_{0}= \pm \omega_{\mathbf{p}}$ are just over the branch cuts since when $|\mathbf{p}|>\kappa$ then $\omega_{\mathbf{p}}>\Omega_{B}$.

$\delta\left(p^{2}+\mu^{2}\right)=\frac{\delta\left(p_{0}-\omega_{\mathbf{p}}^{\mu}\right)+\delta\left(p_{0}+\omega_{\mathbf{p}}^{\mu}\right)}{2 \omega_{\mathbf{p}}^{\mu}}, \quad \omega_{\mathbf{p}}^{\mu}=\sqrt{\mathbf{p}^{2}+\mu^{2}}$,

and the integral over $p_{0}$ in (138) can be trivially carried out

$$
\begin{aligned}
\left.i \Delta_{F}^{\kappa}(x-y)\right|_{B}= & i \int_{-\kappa^{2}}^{+\infty} \frac{d \mu^{2} \kappa}{(2 \pi) \sqrt{\kappa^{2}+\mu^{2}}} \frac{1}{\mu^{2}+m^{2}+i \varepsilon} \\
& \times\left[\int_{|\mathbf{p}|>\kappa} \frac{d^{3} p e^{i \mathbf{p}(\mathbf{x}-\mathbf{y})}}{(2 \pi)^{3} 2 \omega_{\mathbf{p}}^{\mu}}\left(e^{-i \omega_{\mathbf{p}}^{\mu}\left(x_{0}-y_{0}\right)}+e^{i \omega_{\mathbf{p}}^{\mu}\left(x_{0}-y_{0}\right)}\right)\right] .
\end{aligned}
$$

The term in square brackets is just the restriction to $|\mathbf{p}|>\kappa$ of the Feynman propagator $i \Delta_{F}\left(x-y ; \mu^{2}\right)$ minus the antipropagator $i \Delta_{A F}\left(x-y ; \mu^{2}\right)$ or, equivalently, the Hadamard (anticommutator) function $G^{(1)}\left(x-y ; \mu^{2}\right)$ of an ordinary scalar field of mass $\mu$

$$
\begin{aligned}
& \left.G^{(1)}\left(x-y ; \mu^{2}\right)\right|_{B} \\
& \quad=\left.i \Delta_{F}\left(x-y ; \mu^{2}\right)\right|_{B}-\left.i \Delta_{A F}\left(x-y ; \mu^{2}\right)\right|_{B} \\
& \quad=\int_{|\mathbf{p}|>\kappa} \frac{d^{3} p e^{i \mathbf{p}(\mathbf{x}-\mathbf{y})}}{(2 \pi)^{3} 2 \omega_{\mathbf{p}}^{\mu}}\left(e^{-i \omega_{\mathbf{p}}^{\mu}\left|x_{0}-y_{0}\right|}+e^{i \omega_{\mathbf{p}}^{\mu}\left|x_{0}-y_{0}\right|}\right) .
\end{aligned}
$$

We can thus write (140) in terms of a Källen-Lehmann-like spectral representation,

$$
\begin{aligned}
\left.i \Delta_{F}^{\kappa}(x-y)\right|_{B}= & \int_{-\kappa^{2}}^{+\infty} d \mu^{2} \sigma\left(\mu^{2}\right) \frac{1}{2}\left[\left.i \Delta_{F}\left(x-y ; \mu^{2}\right)\right|_{B}\right. \\
& \left.-\left.i \Delta_{A F}\left(x-y ; \mu^{2}\right)\right|_{B}\right]
\end{aligned}
$$


where the spectral function $\sigma\left(\mu^{2}\right)$ is the discontinuity at the cut from $-\kappa^{2}$ to $+\infty$ of the function $1 / f_{\kappa}\left(\mu^{2}\right)=$ $\left(2 \pi i\left(\mu^{2}+m^{2}\right) \sqrt{1+\mu^{2} / \kappa^{2}}\right)^{-1}$

$$
\sigma\left(\mu^{2}\right)=-\frac{2}{2 \pi i} \frac{\kappa}{\sqrt{\kappa^{2}+\mu^{2}}} \frac{1}{\mu^{2}+m^{2}+i \varepsilon} .
$$

The analysis above suggest that the $\kappa$-deformed Feynman propagator for trans-Planckian momenta should take the form of half the Hadamard function,

$$
\begin{aligned}
& \frac{1}{2}\left\langle 0\left|\left\{\phi^{B}(x),\left(\phi^{B}(y)\right)^{*}\right\}\right| 0\right\rangle \\
& \equiv \frac{1}{2}\left[\left\langle 0\left|\phi^{B}(x)\left(\phi^{B}(y)\right)^{*}\right| 0\right\rangle+\left\langle 0\left|\left(\phi^{B}(y)\right)^{*} \phi^{B}(x)\right| 0\right\rangle\right] .
\end{aligned}
$$

In order to check this explicitly, we evaluate the anticommutator of the field in region $B$. For trans-Planckian momenta, the function $f_{\kappa}$ takes the form $f_{\kappa}\left(p_{0}, \mathbf{p}\right)=$ $\frac{2 \pi i}{\kappa} \sqrt{p_{0}^{2}-\Omega_{B}^{2}}\left(p_{0}^{2}-\omega_{\mathbf{p}}^{2}\right)$, where $\Omega_{B}=\sqrt{\mathbf{p}^{2}-\kappa^{2}}$. The singularity structure of $1 / f_{\kappa}$ is shown in Fig. 7. Taking into account Eqs. (118) and (121), we can write the field as

$$
\begin{aligned}
\phi_{\mathbf{p}}^{B}\left(x_{0}\right)= & \frac{1}{2 \pi i} \sum_{i=1}^{2} \int_{\Gamma_{i}^{B}} \frac{d p_{0} \kappa}{\sqrt{p_{0}^{2}-\Omega_{B}^{2}}} \frac{e^{-i p_{0} x_{0}}}{p_{0}^{2}-\omega_{\mathbf{p}}^{2}} a\left(p_{0}, \mathbf{p}\right) \\
& =\int_{\Omega_{B}}^{\infty} d p_{0} \Delta_{\Gamma_{B}}\left[\frac{1}{f_{\kappa}\left(p_{0}, \mathbf{p}\right)}\right] e^{-i p_{0} x_{0}} a\left(p_{0}, \mathbf{p}\right) \\
& +\int_{\Omega_{B}}^{\infty} d p_{0} \Delta_{\Gamma_{B}}\left[\frac{1}{f_{\kappa}\left(p_{0}, \mathbf{p}\right)}\right] e^{i p_{0} x_{0}} a\left(-p_{0}, \mathbf{p}\right),
\end{aligned}
$$

where the discontinuity functional at the cut of $1 / f_{\kappa}$ is

$$
\Delta_{\Gamma_{B}}\left[\frac{1}{f_{\kappa}\left(p_{0}, \mathbf{p}\right)}\right]=-\frac{2}{2 \pi i} \frac{\kappa}{\sqrt{p_{0}^{2}-\Omega_{B}^{2}}} \frac{1}{p_{0}^{2}-\omega_{\mathbf{p}}^{2}} .
$$

Following the procedure already employed in the region $A$, the quantum counterpart of the field (145) and of its complex conjugate, are obtained by promoting the entire analytic functions $a(p)$ and $a(-p)$ to annihilation and creation operators of particles and antiparticles, respectively. The commutation rules for annihilation and creation operators of excitations with four momentum belonging to the cut are then

$$
\begin{aligned}
& {\left.\left[a(p), a^{\dagger}(q)\right]\right|_{p, q \in \Gamma_{B}}=\frac{\delta(p-q)}{\Delta_{\Gamma_{B}}\left[1 / f\left(-p^{2}\right)\right]},} \\
& {\left.\left[b(p), b^{\dagger}(q)\right]\right|_{p, q \in \Gamma_{B}}=\frac{\delta(p-q)}{\Delta_{\Gamma_{B}}\left[1 / f\left(-p^{2}\right)\right]},}
\end{aligned}
$$

with all the others commutators being zero. Using the Eqs. (145)-(147), one can now compute the two-point functions for trans-Planckian momenta as

$$
\begin{aligned}
\left\langle 0\left|\phi^{B}(x)\left(\phi^{B}(y)\right)^{*}\right| 0\right\rangle & =\int_{|\mathbf{p}|,|\mathbf{q}|>\kappa} \frac{d^{3} p d^{3} q}{(2 \pi)^{3}} e^{i \mathbf{p} \cdot \mathbf{x}} e^{-i \mathbf{q} \cdot \mathbf{y}}\left\langle 0\left|\phi_{\mathbf{p}}^{B}\left(x_{0}\right)\left(\phi_{\mathbf{q}}^{B}\left(y_{0}\right)\right)^{*}\right| 0\right\rangle \\
& =\int \frac{d^{3} p}{(2 \pi)^{3}} e^{i \mathbf{p} \cdot(\mathbf{x}-\mathbf{y})}\left(-\frac{2}{2 \pi i} \int_{\Omega_{B}}^{+\infty} \frac{d p_{0} \kappa}{\sqrt{p_{0}^{2}-\Omega_{B}^{2}}} \frac{e^{-i p_{0}\left(x_{0}-y_{0}\right)}}{p_{0}^{2}-\omega_{\mathbf{p}}^{2}}\right), \\
\left\langle 0\left|\left(\phi^{B}(y)\right)^{*} \phi^{B}(x)\right| 0\right\rangle & =\int_{|\mathbf{p}|,|\mathbf{q}|>\kappa} \frac{d^{3} p d^{3} q}{(2 \pi)^{3}} e^{-i \mathbf{p} \cdot \mathbf{x}} e^{i \mathbf{q} \cdot \mathbf{y}}\left\langle 0\left|\left(\phi_{\mathbf{q}}^{B}\left(y_{0}\right)\right)^{*} \phi_{\mathbf{p}}^{B}\left(x_{0}\right)\right| 0\right\rangle \\
& =\int \frac{d^{3} p}{(2 \pi)^{3}} e^{-i \mathbf{p} \cdot(\mathbf{x}-\mathbf{y})}\left(-\frac{2}{2 \pi i} \int_{\Omega_{B}}^{+\infty} \frac{d p_{0} \kappa}{\sqrt{p_{0}^{2}-\Omega_{B}^{2}}} \frac{e^{i p_{0}\left(x_{0}-y_{0}\right)}}{p_{0}^{2}-\omega_{\mathbf{p}}^{2}}\right) .
\end{aligned}
$$

The $p_{0}$ integrals in this last expressions are ill defined since the poles $\pm \omega_{\mathbf{p}}$ are on the path of integration $\left(\omega_{\mathbf{p}}>\Omega_{B}\right)$; however, we can adopt the prescription in the spectral function (143) to avoid the poles, and thus the vacuum expectation value of half the anticommutator of the fields becomes

$$
\frac{1}{2}\left\langle 0\left|\left\{\phi^{B}(x),\left(\phi^{B}(y)\right)^{*}\right\}\right| 0\right\rangle=-\frac{1}{2 \pi i} \int_{|\mathbf{p}|>\kappa} \frac{d^{3} p}{(2 \pi)^{3}} e^{i \mathbf{p} \cdot(\mathbf{x}-\mathbf{y})} \int_{\Omega_{B}}^{+\infty} \frac{d p_{0} \kappa}{\sqrt{p_{0}^{2}-\Omega_{B}^{2}}} \frac{e^{-i p_{0}\left(x_{0}-y_{0}\right)}+e^{i p_{0}\left(x_{0}-y_{0}\right)}}{p_{0}^{2}-\omega_{\mathbf{p}}^{2}+i \varepsilon} .
$$

In this equation, the $p_{0}$ integral is just the integral $\mathcal{I}_{\mathbf{p}}^{B}$ defined in (87). We thus obtain that the Hadamard function of the nonlocal field is equal to the restriction $\left.i \Delta_{F}^{\kappa}(x-y)\right|_{B}$ of the $\kappa$-deformed Feynman propagator to trans-Planckian momenta

$$
\frac{1}{2}\left\langle 0\left|\left\{\phi^{B}(x),\left(\phi^{B}(y)\right)^{*}\right\}\right| 0\right\rangle=\left.i \int_{|\mathbf{p}|>\kappa} \frac{d^{3} p}{(2 \pi)^{4}} e^{i \mathbf{p} \cdot(\mathbf{x}-\mathbf{y})} \mathcal{I}_{\mathbf{p}}^{B}\left(x_{0}-y_{0}\right) \equiv i \Delta_{F}^{\kappa}(x-y)\right|_{B} .
$$


Considering now the relations (86), (135), and (150), we can finally write down the expression of $i \Delta_{F}^{\kappa}(x-y)$ in terms of the vacuum expectation values of the nonlocal scalar fields on ordinary Minkowski space. The $\kappa$-deformed Feynman propagator coincides with the T-product of such nonlocal fields for sub-Planckian momenta and to half their Hadamard function for trans-Planckian momenta

$$
\begin{aligned}
i \Delta_{F}^{\kappa}(x-y)= & \left\langle 0\left|T\left\{\phi^{A}(x)\left(\phi^{A}(y)\right)^{*}\right\}\right| 0\right\rangle \\
& +\frac{1}{2}\left\langle 0\left|\left\{\phi^{B}(x),\left(\phi^{B}(y)\right)^{*}\right\}\right| 0\right\rangle .
\end{aligned}
$$

Let us note that in the limit $\kappa \rightarrow \infty$ the rhs of this equation approaches the time-ordered two-point function as a consequence of the fact that in this limit the region $B$ disappears.

Although the relation (151) may look unusual, since it contains a splitting that depends on the value of the spatial momenta, it turns out that a similar expression for the propagator emerges in an apparently unrelated context, namely for the Feynman propagator of a degenerate Fermi gas (see, e.g., the standard textbook reference [59]). In this case, the "deformation scale" is set by the Fermi momentum $k_{F}$, and the standard Feynman propagator for the Dirac field is recovered in the limit $k_{F} \rightarrow 0$, so that, in particular, the Fermi momentum plays the role of an IR deformation parameter. Indeed, when one replaces the standard vacuum with a noninteracting Fermi gas of electrons at zero temperature with Fermi momentum $k_{F}$, the resulting propagator coincides with the advanced propagator for spatial momenta $\mathbf{p}$ lower than $k_{F}$ and to the ordinary time-ordered two-point function for $|\mathbf{p}|>k_{F}$. A physical interpretation of such a modification can be given by recalling that the standard Feynman propagator corresponds to an advanced propagation of the negative-energy solutions and to a retarded propagation of the positiveenergy solutions. Now, in a degenerate Fermi gas, all the levels in the positive-energy electron continuum are occupied up to the Fermi momentum $k_{F}$. Excitations related to these energy levels have to be treated like negative-energy states, i.e., they propagate backwards in time via the advanced propagator. Accordingly, when we consider states with an energy below the corresponding Fermi energy $E_{F}=\sqrt{k_{F}^{2}+m_{e}^{2}}$, both the negative-energy solutions and the positive-energy solutions propagates in an advanced way, conversely, when $|\mathbf{p}|>k_{F}$, negative-energy solutions still propagate in an advanced way while the positive-energy solutions propagate in a retarded way, so that we recover the standard structure that gives rise to the time-ordered twopoint function.

In the analysis, we presented the deformation parameter $\kappa$, usually identified with the Planck energy $E_{p}$, is instead a UV scale and the $\kappa$-deformed Feynman propagator differs from the time-ordered two-point function for spatial momenta bigger than $\kappa$. Indeed, for trans-Planckian momenta, the $\kappa$-deformed propagator has the form of the Hadamard function which, being the vacuum expectation value of the anticommutator of the field, does not posses a time ordering. Nonetheless, like the T-product, the anticommutator is symmetric under exchange of the spacetime arguments of the fields. A physical interpretation for the appearance of the Hadamard function for trans-Planckian momenta is however less straightforward than the interpretation of the propagator structure of a degenerate Fermi gas. Indeed, we now deal with vacuum expectation value of a nonlocal field, in which both the simple poles and the branch cuts contributes. The expression (151) for the $\kappa$-deformed Feynman propagator, suggests that for trans-Planckian momenta, we lose the notion of time orientation; the two Wightman functions $\left\langle 0\left|\phi^{B}(x)\left(\phi^{B}(y)\right)^{*}\right| 0\right\rangle$ and $\left\langle 0\left|\left(\phi^{B}(y)\right)^{*} \phi^{B}(x)\right| 0\right\rangle$ contribute both for $x_{0}>y_{0}$ and $x_{0}<y_{0}$.

\section{DISCUSSION}

In this work, we provided a comprehensive description of the structure and properties of the Feynman propagator of a $\kappa$-deformed field theory. We started from its derivation from a noncommutative generating functional and described the nontrivial singularity structure determined by the curved geometry of momentum space dual to the $\kappa$-Minkowski space. We showed how such singularity structure is responsible for the new features of the $\kappa$ Feynman propagator, and how such features are intimately related to the different behavior of the propagator for sub and trans-Planckian field modes. Our results showed that the propagation of perturbations in $\kappa$-deformed field theory can be equivalently described in terms of perturbations generated by a fuzzy source, which cannot be sharply localized in spacetime, or by a ordinary source which generates perturbations mediated by a $\kappa$-deformed propagator whose nontrivial spacetime profile was analyzed in detail.

Our analysis also addressed the question of whether the $\kappa$-deformed Feynman propagator derived from the generating functional, which we showed to be a Green's function of the $\kappa$-deformed Klein-Gordon operator, can be related to the vacuum expectation value of suitable products of field operators. In order to explore such a relationship, we resorted to a mapping of noncommutative fields on $\kappa$-Minkowski space to fields on ordinary Minkowski space with a nonlocal kinetic term. Adopting techniques previously developed in the literature for the canonical quantization of nonlocal field theories, we showed that the $\kappa$-deformed Feynman propagator can be related to the vacuum expectation value of products of nonlocal field operators. We obtained that for sub-Planckian field modes the propagator coincides with the vacuum expectation value of the time-ordered product of nonlocal fields, while for trans-Planckian momenta, it is related to the nonlocal Hadamard function, i.e., the vacuum expectation value of the anticommutator of nonlocal field operators. This peculiar behavior is reminiscent of the 
propagation of electrons in a Fermi gas where above and below the Fermi momentum their propagator is described by different kinds of two-point functions. While electrons above the Fermi momentum propagate as ordinary free electrons, excitations below the Fermi level (holes) behave has antiparticles and are described by an advanced propagator. In our case, the deformation parameter $\kappa$ sets a UV momentum scale above which propagation of excitations is described by the Hadamard propagator in which a notion of time ordering is no longer present. This result provides an interesting picture of how the noncommutativity of $\kappa$-Minkowski space affects the propagation of particles in the deep UV and provides valuable insight for understanding the physical properties of this particular noncommutative deformation of quantum field theory.

While the main focus of our analysis has been on the study of $\kappa$-deformed propagation, it would be interesting to further explore the alternative picture of $\kappa$-deformation as ordinary propagation of perturbations from a smeared out source as discussed in Sec. III B. In particular, it should be in principle possible to reformulate this scenario in terms of an equivalent nonlocal field theory on ordinary Minkowski spacetime and investigate its nontrivial UV effects. We postpone such a study to future work.
[1] S. Carlip, Classical Quantum Gravity 34, 193001 (2017).

[2] J. Ambjorn, J. Jurkiewicz, and R. Loll, Phys. Rev. Lett. 95, 171301 (2005).

[3] S. Carlip, Int. J. Mod. Phys. D 25, 1643003 (2016).

[4] O. Lauscher and M. Reuter, J. High Energy Phys. 10 (2005) 050.

[5] P. Horava, Phys. Rev. Lett. 102, 161301 (2009).

[6] D. Benedetti, Phys. Rev. Lett. 102, 111303 (2009).

[7] L. Modesto, Classical Quantum Gravity 26, 242002 (2009).

[8] D. Benedetti and J. Henson, Phys. Rev. D 80, 124036 (2009).

[9] G. Calcagni, J. High Energy Phys. 03 (2010) 120.

[10] G. Calcagni, A. Eichhorn, and F. Saueressig, Phys. Rev. D 87, 124028 (2013).

[11] G. Calcagni, D. Oriti, and J. Thrigen, Phys. Rev. D 91, 084047 (2015).

[12] T. Padmanabhan, S. Chakraborty, and D. Kothawala, Gen. Relativ. Gravit. 48, 55 (2016).

[13] S. Carlip, Classical Quantum Gravity 32, 232001 (2015).

[14] T. P. Sotiriou, M. Visser, and S. Weinfurtner, Phys. Rev. D 84, 104018 (2011).

[15] G. Amelino-Camelia, M. Arzano, G. Gubitosi, and J. Magueijo, Phys. Lett. B 736, 317 (2014).

[16] S. Majid and H. Ruegg, Phys. Lett. B 334, 348 (1994).

[17] M. Arzano and T. Trzesniewski, Phys. Rev. D 89, 124024 (2014).

[18] J. Kowalski-Glikman and S. Nowak, arXiv:hep-th/0411154.

[19] J. Lukierski, H. Ruegg, A. Nowicki, and V. N. Tolstoi, Phys. Lett. B 264, 331 (1991).

[20] J. Lukierski, A. Nowicki, and H. Ruegg, Phys. Lett. B 293, 344 (1992).

[21] G. Amelino-Camelia, M. Arzano, G. Gubitosi, and J. Magueijo, Phys. Rev. D 88, 103524 (2013).

[22] G. Amelino-Camelia, F. Brighenti, G. Gubitosi, and G. Santos, Phys. Lett. B 767, 48 (2017).

[23] K. Nozari, V. Hosseinzadeh, and M. A. Gorji, Phys. Lett. B 750, 218 (2015).

[24] V. Husain, S. S. Seahra, and E. J. Webster, Phys. Rev. D 88, 024014 (2013).

[25] N. Alkofer, G. D’Odorico, F. Saueressig, and F. Versteegen, Phys. Rev. D 94, 104055 (2016).
[26] M. Arzano and G. Calcagni, Eur. Phys. J. C 77, 835 (2017).

[27] M. Arzano and G. Calcagni, Int. J. Mod. Phys. D 26, 1743030 (2017).

[28] M. Arzano and J. Kowalski-Glikman, Phys. Lett. B 771, 222 (2017).

[29] G. Amelino-Camelia and M. Arzano, Phys. Rev. D 65, 084044 (2002).

[30] M. Arzano, Phys. Rev. D 83, 025025 (2011).

[31] L. Freidel, J. Kowalski-Glikman, and S. Nowak, Phys. Lett. B 648, 70 (2007).

[32] J. Kowalski-Glikman and A. Walkus, Mod. Phys. Lett. A 24, 2243 (2009).

[33] M. Arzano, G. Gubitosi, J. Magueijo, and G. AmelinoCamelia, Phys. Rev. D 92, 024028 (2015).

[34] J. Fuchs and C. Schweigert, Symmetries, Lie Algebras and Representations: A Graduate Course for Physicists (Cambridge University Press, Cambridge, 1997).

[35] N. R. Bruno, G. Amelino-Camelia, and J. KowalskiGlikman, Phys. Lett. B 522, 133 (2001).

[36] G. Amelino-Camelia, Phys. Lett. B 510, 255 (2001).

[37] G. Amelino-Camelia, Int. J. Mod. Phys. D 11, 35 (2002).

[38] G. Amelino-Camelia, Symmetry 2, 230 (2010).

[39] J. Kowalski-Glikman and S. Nowak, Phys. Lett. B 539, 126 (2002).

[40] A. Agostini, G. Amelino-Camelia, and M. Arzano, Classical Quantum Gravity 21, 2179 (2004).

[41] J. Magueijo and L. Smolin, Phys. Rev. Lett. 88, 190403 (2002).

[42] S. Hossenfelder, Living Rev. Relativity 16, 2 (2013).

[43] P. Kosinski, J. Lukierski, P. Maslanka, and J. Sobczyk, Mod. Phys. Lett. A 10, 2599 (1995).

[44] A. Borowiec and A. Pachol, J. Phys. A 43, 045203 (2010).

[45] J. Lukierski, H. Ruegg, and V. N. Tolstoi, Quantum kappa Poincare 1994 (unpublished).

[46] G. Amelino-Camelia, Living Rev. Relativity 16, 5 (2013).

[47] G. Amelino-Camelia and L. Smolin, Phys. Rev. D 80, 084017 (2009).

[48] S. Giller, C. Gonera, P. Kosinski, and P. Maslanka, arXiv: q-alg/9602006.

[49] P. Kosinski, P. Maslanka, and J. Sobczyk, Czech. J. Phys. 46, 201 (1996). 
[50] A. Sitarz, Phys. Lett. B 349, 42 (1995).

[51] A. Agostini, G. Amelino-Camelia, M. Arzano, A. Marciano, and R. A. Tacchi, Mod. Phys. Lett. A 22, 1779 (2007).

[52] L. Freidel, J. Kowalski-Glikman, and S. Nowak, Int. J. Mod. Phys. A 23, 2687 (2008).

[53] C. Guedes, D. Oriti, and M. Raasakka, J. Math. Phys. (N.Y.) 54, 083508 (2013).

[54] M. Arzano, D. Latini, and M. Lotito, SIGMA 10, 079 (2014).

[55] M. Arzano, J. Kowalski-Glikman, and A. Walkus, Classical Quantum Gravity 27, 025012 (2010).

[56] L. Freidel and T. Rempel, arXiv:1312.3674.

[57] G. Gubitosi, M. Arzano, and J. Magueijo, Phys. Rev. D 93, 065027 (2016).

[58] M. Arzano, G. Gubitosi, J. Magueijo, and G. AmelinoCamelia, Phys. Rev. D 91, 125031 (2015).

[59] W. Greiner and J. Reinhart, Quantum Electrodynamics (Springer, Berlin, Heidelberg, 1992).

[60] P. Kosinski, J. Lukierski, and P. Maslanka, Phys. Rev. D 62 , 025004 (2000).

[61] P. Kosinski, P. Maslanka, J. Lukierski, and A. Sitarz, arXiv: hep-th/0307038.

[62] F. Mercati and M. Sergola, Phys. Rev. D 98, 045017 (2018).

[63] M. Arzano and A. Marciano, Phys. Rev. D 76, 125005 (2007).
[64] M. Arzano and D. Benedetti, Int. J. Mod. Phys. A 24, 4623 (2009).

[65] G. Amelino-Camelia, L. Freidel, J. Kowalski-Glikman, and L. Smolin, Phys. Rev. D 84, 084010 (2011).

[66] M. Arzano and J. Kowalski-Glikman, Classical Quantum Gravity 28, 105009 (2011).

[67] G. Amelino-Camelia, M. Arzano, J. Kowalski-Glikman, G. Rosati, and G. Trevisan, Classical Quantum Gravity 29, 075007 (2012).

[68] L. Freidel, R. G. Leigh, and D. Minic, J. High Energy Phys. 06 (2015) 006.

[69] M. Arzano, Phys. Rev. D 77, 025013 (2008).

[70] K. S. Stelle, Phys. Rev. D 16, 953 (1977).

[71] J. Z. Simon, Phys. Rev. D 41, 3720 (1990).

[72] R. P. Woodard, Scholarpedia 10, 32243 (2015).

[73] R. L. P. G. do Amaral and E. C. Marino, J. Phys. A 25, 5183 (1992).

[74] D. G. Barci, L. E. Oxman, and M. Rocca, Int. J. Mod. Phys. A 11, 2111 (1996).

[75] D. G. Barci, C. G. Bollini, L. E. Oxman, and M. C. Rocca, arXiv:hep-th/9606183.

[76] A. Belenchia, D. M. T. Benincasa, and S. Liberati, J. High Energy Phys. 03 (2015) 036. 